\begin{tabular}{|l||c||c|c|c|c|c||c|}
\hline Component & GFAP & S100 & Keratin & Desmin & SMA & CD34 & CD10 \\
\hline \hline Glial & $100 \%$ & $100 \%$ & $0 \%$ & $62 \%$ & $50 \%$ & $62 \%$ & $0 \%$ \\
\hline \hline Sarcomatous & $0 \%$ & $12.5 \%$ & $0 \%$ & $50 \%$ & $100 \%$ & $0 \%$ & $100 \%$ \\
\hline
\end{tabular}

\title{
Pancreas and Biliary Tree
}

Conclusions: To our knowledge, this study represents the first to describe CD10 expression in gliosarcomas. While negative in the glial components, CD10 always was positive in the sarcomatous components. Therefore, $\mathrm{CD} 10$ may be a useful marker for histologic delineation of sarcomatous elements of gliosarcomas. Whether or not $\mathrm{CD} 10$ plays a biologic role in gliosarcomas, akin to that in a number of other malignant neoplasms, remains to be elucidated.

1752 Immunohistochemical and Gene Expression Analysis of Recurrent Glioblastoma Shows Increased Expression of Mesenchymal Markers on Tumor Recurrence

Matthew Wood, Gerald Reis, Joanna Phillips. University of California, San Francisco, CA.

Background: Glioblastoma (astrocytoma, WHO grade IV) is characterized by molecular heterogeneity, near universal tumor recurrence, and a poor clinical outcome despite the use of multiple therapeutic modalities. The stability of glioblastoma molecular traits on recurrence is only partly characterized, and additional data are needed to guide treatment of recurrent disease and to identify drivers of heterogeneity in recurrent tumors. Design: We generated tissue microarrays of paired primary and recurrent glioblastoma specimens ( $\mathrm{N}=21$ pairs) for immunohistochemical ( $\mathrm{IHC}$ ) analysis, and simultaneously extracted messenger RNA (mRNA) from a subset of paired tumors to correlate gene expression changes with IHC findings. Messenger RNA levels for a panel of 173 genes were assessed using Nanostring technology. The IHC markers included Ki67, CD34, phosphorylated S6 kinase (pS6K), YKL40, phosphorylated STAT3 (pSTAT3), CD44, OLIG2, EGFR, and IDH1 ${ }^{\mathrm{R} 132 \mathrm{H}}$. Protein expression levels on IHC were scored on a 3-unit semi-quantitative scale; CD44 was scored on a 6-unit scale.

Results: Recurrent glioblastoma showed a significant increase in protein expression of the mesenchymal marker CD44 (4.34 versus 3.42 units, $\mathrm{p}<0.043$ ), which was accompanied by an increase in CD44 mRNA $(\mathrm{p}<0.028)$ and reduced mRNA levels of the proneural marker OLIG2 $(\mathrm{p}<0.004)$. Primary tumors with low protein expression of mesenchymal markers CD44, YKL40 or pSTAT3 showed increased expression of these markers on tumor recurrence (mean increase 2.7 units [p $<0.0001], 0.41$ units [p $<0.0136$ ], and 0.89 units [p $<0.0058]$, respectively). Primary tumors with high protein expression of CD44 and YKL40 showed persistently high expression on recurrence. Conversely, primary tumors with high protein expression OLIG2 showed reduced OLIG2 expression on tumor recurrence (mean decrease 1.57 units, $p<0.0214$ ), while primary tumors with low OLIG2 levels showed no significant change on recurrence. Conclusions: Recurrent glioblastoma showed increased expression of markers associated with a mesenchymal phenotype by both gene expression and immunohistochemical analysis. The mechanisms driving this process may give insight into the molecular evolution of glioblastoma in response to treatment.

\section{Discarded Casette Content From Routine Vitrectomies Includes} Tissue That Provide Valuable Information in Diabetic Patients

Pablo Zoroquiain, Sarah Alghamdi, Natalia Vila, Vasco Bravo-Filho, Patrick Logan, Michael Kapusta, John Chen, Miguel Burnier. McGill University, Montreal, QC, Canada.

Background: Diabetic retinopathy is a clinical diagnosis made by fundus examination. When it comes to diabetes progression, it is not possible to obtain a biopsy in the eye where the risks are higher than the benefits. Our purpose is to describe the histopathological features of vitreous samples from diabetic patients.

Design: Vitreous specimens from 137 patients who underwent pars planavitrectomy for different clinical conditions were analyzed.All samples were centrifuged and the resulting pellet from each sample was fixed in a 1:1 alcohol and formalin solution. The supernatant was discarded and sediment was processed as part of routine paraffin section histopathology. The sample characteristics, cell type and vascular findings were evaluated and categorized ( $0=$ negative; $1=$ low; $2=$ high). When vessels were recorded, the presence of pericytes was evaluated by alpha-SMA(aSMA), and was semiquantified as total loss (3), $>50 \%(2),<50 \%(1)$ and no loss $(0)$. The results were compared between diabetic and non-diabetic patients.

Results: Of the 137 patients included only 12 samples were excluded in this study due to scant material. Of the 125 included(58 diabetic, 67 non-diabetic), 75 were male. The mean age of included patients was $64.2+14$ years. The presence of haemorrhage, inflammatory cells and histiocytes were significantly higher in the diabetic group than the non-diabetic group ( $P=0.001 ; P=0.028 ; P=0.016$ respectively). Moreover, the diabetic group had significantly more vessels $(P=0.001)$ and ghost vessels $(P=0.049)$. All non diabetic cases had a 0 aSMA score while all diabetic ones showed pericyte loss ( $>1$ score).

Conclusions: In this study, we found different histopathological features in vitreous samples from diabetic and non-diabetic patients. The presence of haemorrhage, histiocytes, vessels and ghost vessels were associated with a diagnosis of diabetes. Analysis of vitreous cytology may serve as a diagnostic tool for clinically undetectable diabetic retinopathy. In addition, this technique may be used to evaluate diabetic retinopathy-like damage in glucose intolerant patients.

1754 Gallbladder Carcinomas With Sarcomatoid Component: Clinicopathologic Analysis of 11 Cases

Gizem Akkas, Bahar Memis, Pelin Bagci, Nevra Dursun, Burcu Saka, Olca Basturk, Kee-Taek Jang, Juan Roa, Juan Sarmiento, Volkan Adsay. EU, Atlanta, GA; Wayne State University, Detroit, MI; SMC, Seoul, Korea; PCU, Santiago, Chile.

Background: Sarcomatoid differentiation is uncommon in gallbladder carcinomas (GBC), and data on the clinicopathologic characteristics of GBCs with a sarcomatoid component is highly limited.

Design: Among $656 \mathrm{GBCs}, 11$ cases with a sarcomatoid component constituting ${ }^{3} 25 \%$ of the tumor were identified. Their clinicopathologic characteristics were analyzed/ contrasted with those of ordinary GBC (O-GBC).

Results: The patients were 9 female, 2 male ( $\mathrm{F} / \mathrm{M}=4.5$, vs 3.9 in $\mathrm{O}-\mathrm{GBC})$. Mean age was $71 \mathrm{yr}$ (vs 64). Mean and median tumor sizes were 5.3 and $4.6 \mathrm{~cm}$ (vs 2.9 and 2.5, respectively; $\mathrm{P}=0.01)$. Nine patients $(84 \%)$ presented with advanced stage (pT3/4) tumors (vs 48\%). Of 7 known, 6 had gallstones. Microscopically, an adenocarcinoma component was present in 9 , constituting $1-75 \%$ of the tumor, and 8 had surface dysplasia/CIS. An intracholecystic papillary tubular neoplasm was identified in 1 . Seven revealed pleomorphic-sarcomatoid pattern (as defined in the upper aerodigestive tract), 4 showed prominent spindle cell pattern composed of subtle, bland, stromal-appearing cells, and 1 was mistaken as granulation tissue on frozen section. Three cases had angiosarcomatoid pattern with prominent clefts and/or intermixed vascularity with RBCs. Two cases had heterologous elements ( 1 bone/cartilage, 1 cartilage only). Only 1 showed focal osteoclast-like giant cells. Lymph node metastasis was present in $2 / 5$. Immunohistochemically, vimentin was $(+)$ in $6 / 6$, epithelial markers were retained in the sarcomatoid components of most (AE1/AE3 6/7, CK18 5/6, and EMA in 5/6) along with at least focal expression of MUC1 (5/6). p63 was $(+)$ in 2/6. Actin, desmin and $\mathrm{S} 100$ were negative in all (0/6). Median Ki67 index was 40\%. P53 expression was $>60 \%$ in $6 / 6$. MLH1 and MSH2 were retained. One patient died peri-operatively. Of the remaining 10,8 died of disease within 3 to $8 \mathrm{mos}$, and 2 were alive at 9 and 15 mos (vs median survival of 26 mos for O-GBC).

Conclusions: Sarcomatoid undifferentiated component is seen in $<2 \%$ of GBC. It typically occurs in the company of ordinary adenocarcinoma. Unlike the ones in the remainder of pancretobiliary tract, these are seldom of "osteoclastic" type. Also, in contrast with the sarcomatoid carcinomas of other sites, these often retain keratin positivity. The patients present with large/advanced-stage tumors that have high proliferation index. Limited data suggests that these tumors are highly aggressive with rapid mortality.

1755 Pathologic Diagnosis as the Reason for Wide Discrepancies in the Literature Regarding the Incidence and Behavior of T1 Gallbladder Cancer (GBC): An Analysis of $\mathbf{4 7 3}$ GBC and Comparison With Literature

Gizem Akkas, Bahar Memis, Olca Basturk, Brian Quigley, Selda Karaaslan, Burcu Saka, Nevra Dursun, Pelin Bagci, Serdar Balci, Juan Sarmiento, Sudeshna Bandyopadhyay, Oscar Escalona, Michael Goodman, Jessica Knight, Alyssa Krasinskas, Juan Roa, Volkan Adsay. EU, Atlanta, GA; Wayne State University, Detroit, MI; PUC, Santiago, Chile.

Background: For T1 GBC, there is highly conflicting data on both its relative frequency among invasive ca $(20-44 \%)$ and prognosis (5-yr, $~ 90 \%$ in Korea/Japan, where cholecystectomy is regarded curative, vs $<50 \%$ in the SEER/West-Other, with radical operations performed).

Design: 473 in-situ and invasive GBC (248 from Chile, 225, US) were carefully re-analyzed.

Results: 124 were determined to be Tis (non-invasive) and 349 invasive. See table for T-stage distribution of invasive ones.

\begin{tabular}{||l||l||l||l||l||}
\hline \hline & PT1 & PT2 & PT3 & TOTAL \\
\hline \hline USA & 7 & 81 & 115 & 203 \\
\hline \hline CHILI & 14 & 84 & 48 & 146 \\
\hline
\end{tabular}

USA: Only 10 had been reported as T1,3 of which proved to be T2. Chile: Of the 51 cases that had been classified as "early GBC" (Tis/T1), 4 was upstaged to T2 and only 14 showed definitive $\mathrm{T} 1$ by the Western criteria. Of the $26 \mathrm{~T} 1$ cases (including 5 consultation cases not included in the frequency analysis), 2 died perioperatively, 2 died of GBC at 6 and $27 \mathrm{mos}, 3$ died of other causes, 2 were lost to follow up, and the remaining 17 were alive without disease at a median of 110 mos (over all 5-year survival, $67 \%$; disease-specific survival, $91 \%$ ).

Conclusions: In Chile, where it has been managed as a major healthcare problem, GBCs are detected in earlier stages (Tis $+\mathrm{T} 1$ constituting $47 \%$ of the cases, vs $12 \%$ in the US). I. With careful pathologic examination and invasiveness criteria employed in the West, $<10 \%$ of invasive GBCs qualify as T1 (US, $3 \%$, Chile $9 \%$ ), which is not surprising considering T1 of GBC essentially corresponds to "intramucosal adenoca" of other sites because of the mucosal thinness, and the tunica muscularis acting like muscularis-mucosa. II. Markedly higher frequency of T1 in the literature (SEER, $40 \%$, Korea, $35 \%$ ) is attributable to the pathologic over-staging of complex Tis cases as $\mathrm{T} 1$, which is also not surprising considering GB mucosa is often complex with florid glandular proliferations and invaginations with pseudoinvasive patterns. III. The behavior of T1 seems to be much better than reported in the West/SEER data(5$\mathrm{yr}, 35-50 \%$ ), which is attributable to under-staged T2s (in this study, 7 that had been reported as T1/EGBC were re-classified as T2). IV. Considering all these challenges/ 
controversies in T-staging, it is advisable to adopt a modification of the staging system already in use in high-incidence regions: "Early" (Tis/T1) versus "locally advanced" (T2 and GB-confined-T3) and "extra-cholecystic spread".

1756 DOG1 Is Not Significantly Expressed By Pancreatic Acinar Cell Carcinomas or Solid-Pseudopapillary Neoplasms, While It Is Often Expressed By Serous Tumors

Deema Alkapalan, Edward Stelow, Charles Quick, Jason Hornick, Andrew Bellizzi. University of Iowa, Iowa City, IA; University of Virginia, Charlottesville, VA; Brigham \& Women's Hospital, Boston, MA; University of Arkansas for Medical Sciences, Little Rock, AR.

Background: Discovered on GIST1 (DOG1), a calcium-activated chloride channel, is most familiar as a gastrointestinal stromal tumor marker. Despite the fact that DOG1 mRNA is highly expressed in pancreatic tissue, DOG1 protein expression has received relatively little attention in this organ, and the few existing immunohistochemistry (IHC) studies are conflicting. In one, DOG1 was advanced as a marker of solid-pseudopapillary neoplasm (SPN) and centroacinar cells, while in another DOG1 was neither expressed in SPN nor serous microcystic adenoma (SMA; a tumor of centroacinar phenotype) Recently, DOG1 was shown to be a sensitive marker of salivary acinic cell carcinoma; pancreatic acinar cell carcinoma (ACC) has not been studied to date.

Design: DOG1 IHC was performed on 17 ACCs, 20 SMAs, and 22 SPNs, using a polyclonal (Spring) and two monoclonal (DOG1.1, Zeta; K9, Novocastra) antibodies. Cases were assessed for extent $(\%)$ and intensity $(0,1+, 2+, 3+)$ of staining and an $\mathrm{H}$-score (extent $\mathrm{x}$ intensity) calculated. Attention was also paid to the pattern of staining (apical, granular/perinuclear, diffuse cytoplasmic). Staining in background pancreas was also noted

Results: One ACC stained with each of the monoclonal antibodies (MoAbs), while 4 cases were positive with the polyclonal antibody (PoAb). Staining with the K9 clone was apical, while staining with the others was granular/perinuclear. Most SMAs demonstrated DOG1 apical staining of varying intensity, with the K9 clone less sensitive. Two SPNs demonstrated diffuse cytoplasmic staining with the PoAb, which appears to be artifactual, as no SPNs stained with the MoAbs. The entire ductal system, from centroacinar cells to the main pancreatic duct demonstrated patchy apical staining of varying intensity $(1-3+)$. Detailed expression data are presented in the Table.

\begin{tabular}{|l||l||l||l|l||l||l||}
\hline \multicolumn{1}{|l||}{} & \multicolumn{2}{c||}{ Polyclonal } & \multicolumn{2}{c||}{ Clone K9 } & \multicolumn{2}{c||}{ Clone DOG1.1 } \\
\hline \hline $\begin{array}{l}\text { Tumor } \\
\text { Type }\end{array}$ & $\begin{array}{l}\text { Positive } \\
\text { Staining }\end{array}$ & $\begin{array}{l}\text { H-Score } \\
\text { Range }\end{array}$ & $\begin{array}{l}\text { Positive } \\
\text { Staining }\end{array}$ & $\begin{array}{l}\text { H-Score } \\
\text { Range }\end{array}$ & $\begin{array}{l}\text { Positive } \\
\text { Staining }\end{array}$ & $\begin{array}{l}\text { H-Score } \\
\text { Range }\end{array}$ \\
\hline \hline ACC & $4 / 17(24 \%)$ & $2-150$ & $1 / 17(6 \%)$ & 10 & $1 / 17(6 \%)$ & 15 \\
\hline \hline SMA & $10 / 12(83 \%)$ & $5-240$ & $16 / 20(80 \%)$ & $5-120$ & $8 / 20(40 \%)$ & $1-80$ \\
\hline \hline SPN & $2 / 22(9 \%)$ & $100-240$ & $0 / 22(0 \%)$ & NA & $0 / 22(0 \%)$ & NA \\
\hline \hline
\end{tabular}

Conclusions: ACCs, unlike salivary acinic cell carcinomas, do not express DOG1 to any significant extent. An earlier report of DOG1-positivity in SPNs likely represents non-specific staining. SMAs express DOG1, reflecting their ductal phenotype.

1757 The Value of Arginase-1 Immunohistochemical Stain in Differentiating Hepatocellular Carcinoma and Metastatic Pancreas Acinar Cell Carcinoma as Compared To Glypican-3

Gokce Askan, Jinru Shia, Volkan Adsay, Carlie Sigel, David Klimstra, Ecmel IsikKaygusuz, Olca Basturk. Memorial Sloan Kettering Cancer Center, New York, NY; Emory University, Atlanta, GA; Zeynep Kamil Hospital, Istanbul, Turkey.

Background: Glypican-3 and relatively newly identified arginase-1, effective immunohistochemical markers for identifying hepatocellular differentiation, have been widely used to diagnose hepatocellular carcinoma (HCC) and to distinguish it from metastatic carcinomas. However, glypican-3 has recently been reported to be also expressed by more than $50 \%$ of pancreatic acinar cell carcinomas (ACCs) (PMID: 23079207). This creates a potential pitfall when evaluating tumors involving the liver as ACCs commonly metastasize to the liver. In this study, we explored the possibility of arginase-1 expression in ACCs.

Design: A tissue microarray (TMA) of pancreatic ACC was created using 3 punches of $0.6 \mathrm{~mm}$ tumor for each case $(\mathrm{n}=28)$. The TMA comprised 11 pure and 17 mixed ACCs [11 mixed acinar-neuroendocrine (MANEC), 4 mixed acinar-ductal (MADC), and 2 mixed acinar-neuroendocrine-ductal carcinoma (MANEDC)]. Acinar and neuroendocrine differentiations were previously confirmed by immunohistochemical labeling for trypsin and/or chymotrypsin and chromogranin and/or synaptophysin, respectively. Glypican-3 and arginase-1 immunohistochemical stains were performed on the TMA. If there was any positivity, the markers were repeated, along with HepPar-1, on a full-faced section of a representative tumor block. Strength and distribution of staining were evaluated.

Results: None of the ACCs expressed arginase-1 but glypican-3 was positive (strong patchy to diffuse) in 7/28 (25\%) of ACCs on both TMA and full-faced sections. One of the 7 glypican-3 positive MANECs was also labeled with HepPar-1 (strong/patchy) and another one revealed a few scattered HepPar-1 positive cells on full-faced sections.

\begin{tabular}{|l||l||l||l||l|l||}
\hline & $\begin{array}{l}\text { Pure ACC } \\
(\mathrm{n}=11)\end{array}$ & $\begin{array}{l}\text { MANEC } \\
(\mathrm{n}=11)\end{array}$ & $\begin{array}{l}\text { MADC } \\
(\mathrm{n}=4)\end{array}$ & $\begin{array}{l}\text { MANEDC } \\
(\mathrm{n}=2)\end{array}$ & $\begin{array}{l}\text { Total } \\
(\mathrm{n}=28)\end{array}$ \\
\hline \hline Arginase-1 & 0 & 0 & 0 & 0 & 0 \\
\hline \hline Glypican-3 & $\begin{array}{l}1 \text { diffuse1 } \\
\text { patchy }\end{array}$ & $\begin{array}{l}2 \text { diffuse2 } \\
\text { patchy }\end{array}$ & 1 patchy & 0 & $7(25 \%)$ \\
\hline \hline HepPar-1 & 0 & $\begin{array}{l}1^{*} \text { patchy }{ }^{*} \\
\text { few cells }\end{array}$ & 0 & 0 & $2(7 \%)$ \\
\hline
\end{tabular}

*The case labeled with both Glypican-3 and HepPar-1.
Conclusions: Although glypican-3 may be positive, arginase-1 appears to be uniformly negative in ACCs. Thus, its use may improve the accuracy in distinguishing hepatocellular carcinoma from metastatic acinar cell carcinoma.

1758 Intraductal Oncocytic Papillary Neoplasm of the Pancreas Have Distinct Molecular Characteristics Than Intraductal Papillary Mucinous Neoplasm

Olca Basturk, Umesh Bhanot, Michael Berger, Peter Allen, Marcus Tan, Sasinya Scott, Ronak Shah, Volkan Adsay, Gokce Askan, David Klimstra. Memorial Sloan Kettering Cancer Center, New York, NY; University of South Alabama, Alabama, AL; Emory University, Atlanta, GA.

Background: In 2010, the WHO reclassified the entity originally described as intraductal oncocytic papillary neoplasm as the "oncocytic-type" of intraductal papillary mucinous neoplasm (IPMN). Although several key molecular alterations of other IPMN types have been discovered, including common mutations in $K R A S, G N A S$, and RNF3, those of oncocytic-type have not been well characterized.

Design: Eleven cases with features of oncocytic-type IPMN were identified. Of these, 9 revealed the entity-defining morphology of arborizing papillae lined by $2-5$ layers of cuboidal cells with oncocytic cytoplasm and prominent, eccentric nucleoli as well as intraepithelial lumina. Of the other 2 cases, 1 had an unusual morphology characterized by a flat lining despite oncocytic cytology. The other was a predominantly pancreatobiliary-type IPMN with a distinct focus of oncocytic-type epithelium, and both components of this case were analyzed separately. For comparison, 2 biliary (intrahepatic bile duct) oncocytic papillary neoplasms were also analyzed. All specimens were microdissected, and deep coverage, targeted next-generation sequencing of 300 key cancer-associated genes was performed.

Results: Among pancreatic neoplasms, the number of mutations per case ranged from 1 to 10 (median=4). There were four repeatedly mutated genes, each in 2 of 9 typical oncocytic-type cases: ARHGAP26, ASXL1, EPHA8, and ERBB4. None of these 9 cases had KRAS or GNAS mutations and only one had RNF43 and PIK3RI mutations. In the case with a flat lining, the only mutation was $K R A S$ (the only $K R A S$-mutated case in the study). However, the one with both pancreatobiliary and oncocytic foci manifested TP53, GNAS and RNF43 mutations in both components and did not reveal any of the 4 mutations detected in the typical oncocytic-type cases. Thus, molecularly, it was similar to other IPMN subtypes. In the 2 biliary cases analyzed, the number of mutations per case was 8 and 4 . The neoplasms did not have those 4 mutations, instead both revealed GNAS mutation, and one also had a PIK3CB mutation.

Conclusions: This small series suggests that when defined strictly, oncocytic-type IPMN has distinct molecular features compared to other types of IPMN and thus should be recognized separately.

1759 Pathologic Evaluation and Reporting of Intraductal Papillary Mucinous Neoplasms of the Pancreas: Recommendations of Verona Consensus Meeting

Olca Basturk, Mari Mino-Kenudson, T Furukawa, G Zamboni, G Marchegiani, C Bassi, R Salvia, G Malleo, S Paiella, Christopher Wolfgang, H Matthaei, G Offerhaus, M Adham, M Bruno, M Reid, A Krasinskas, G Kloppel, N Ohike, T Tajiri, KT Jang, Juan Roa, P Allen, C Fernandez-del Castillo, JY Jang, D Klimstra, R Hruban, Volkan Adsay. Memorial Sloan Kettering Cancer Center, New York, NY; MGH, Boston, MA; Tokyo Women's Medical University, Tokyo, Japan; University of Verona, Verona, Italy; Johns Hopkins University, Baltimore, MD; University of Bonn, Bonn, Germany; University Medical Center, Utrecht, Netherlands; E. Herriot Hosp, Lyon, France; Erasmus University Medical Center, Rotterdam, Netherlands; EU, Atlanta, GA; Tech U, Munchen, Germany; Showa, Yokohama, Japan; Tokai, Tokyo, Japan; SMC, Seoul, Korea; Pontificia U, Santiago, Chile; SNU, Seoul, Korea.

Background: There are no established guidelines for pathologic diagnosis and reporting of intraductal papillary mucinous neoplasms (IPMN).

Design: An international multidisciplinary group brought together by the Veron Pancreas Group in Italy, in 2013, was tasked to identify the problems and devise recommendations.

Results: Recommendations: 1)Crucial to rule out invasive carcinoma with extensive (if not complete) sampling, including uninvolved pancreas. 2)Invasive component is to be documented in a full cancer synoptic report including its size, type (colloid/tubular/ others), grade, stage. 3)The term "minimally invasive" should be avoided; instead invasion size, along with stage and substaging of T1 ( $1 \mathrm{a}, \mathrm{b}, \mathrm{c} ; £ 0.5,>0.5-£ 1$, and $>1$ $\mathrm{cm}$ ), is to be documented. 4)Largest diameter of the invasion, not the distance from the nearest duct, is to be used. 5)A category of "indeterminate (suspicious) for invasion" is acceptable for rare cases. 6)The term "malignant" IPMN should be avoided. 7)The highest grade of dysplasia in the non-invasive component is to be documented separately. 8)Lesion size is to be correlated with imaging findings in cysts with possible rupture. 9) The main duct diameter, and if possible, its involvement is to be documented; however, it is not required to provide main vs branch-duct classification in the report of resected tumor. 10)Subtyping as gastric, intestinal, pancreatobiliary, oncocytic, or mixed is of value. 11)Frozen section is to be performed highly selectively and with appreciation of its shortcomings. 12)In reporting FNAs, it is recommended that the grade of atypia (low vs high) be documented. 13)These principles also apply to other similar tumoral intraepithelial neoplasms (mucinous cystic neoplasms, intra-ampullary, intra-biliary, and intra-cholecystic).

Conclusions: These recommendations will ensure not only proper communication of salient tumor characteristics to the management teams, but also better understanding of these neoplasms, accurate comparison of data between analyses, and development of more effective management algorithms. 
1760 Revised Recommendations for Assessing Precursor Lesions in the Pancreas

Olca Basturk, Seung-Mo Hong, Laura Wood, Volkan Adsay, Jorge Albores-Saavedra, Andrew Biankin, Lodewijk Brosens, Noriyoshi Fukushima, Michael Goggins, Ralph Hruban, Yo Kato, Scott Kern, David Klimstra, Gunter Kloppel, Alyssa Krasinskas, Daniel Longnecker, Anirban Maitra, Hanno Matthei, G Johan Offerhaus, Michio Shimizu, Kyoichi Takaori, Benoit Terris, Shinichi Yachida, Irene Esposito, Toru Furukawa. Memorial Sloan Kettering Cancer Center, New York, NY; Asan Medical Center, Seoul, Korea; Johns Hopkins University, Baltimore, MD; Emory University, Atlanta, GA; Medica Sur Clinic, Mexico, Mexico; University of Glasgow, Glasgow, United Kingdom; University Medical Center, Utrecht, Netherlands; Jichi Medical University, Shimotsuke, Japan; Japanese Foundation for Cancer Research, Tokyo, Japan; TU-Munich, Munich, Germany; Dartmouth-Hitchcock Medical Center, Lebanon, NH; University of Texas MD Anderson Cancer Center, Houston, TX; University of Bonn, Bonn, Germany; Saitama Medical University, Saitama, Japan; Kyoto University, Kyoto, Japan; Hôpital Cochin, Paris, France; National CC, Tokyo, Japan; Tokyo Women's Medical University, Tokyo, Japan.

Background: Recent advances have influenced our approach to the diagnosis and management of pancreatic cancer precursors.

Design: International experts met in June 2014 to discuss recent advances and to revise the 2004 recommendations for assessing pancreatic intraepithelial neoplasia (PanIN), intraductal papillary mucinous neoplasm (IPMN) and mucinous cystic neoplasm (MCN). Results: Consensus recommendations included: 1)To improve concordance and to align with practical consequences, a two-tiered system (low vs high-grade) is proposed for all pancreatic precursor lesions, with the provision that the current PanIN-2 and intermediate-grade dysplasia are now categorized as low-grade. Thus, "high-grade dysplasia" (also designated "carcinoma in-situ" in some places) is to be reserved for only the uppermost end of the spectrum. 2)Intraductal lesions $0.5-1 \mathrm{~cm}$ can be either large PanINs or small (incipient) IPMNs. The term "incipient IPMN" should be reserved for lesions with long finger-like papillae, intestinal-type mucin or GNAS mutations. 3)Additional data are needed to determine if dysplasia at a margin in IPMNs lacking invasive carcinoma predict survival. Therefore, it is recommended to grade dysplasia at a margin even if PanIN cannot be distinguished from IPMN. 4)Conceptually, invasive carcinoma associated with an IPMN is genetically related to the IPMN; concomitant (independent) invasive carcinomas should be genetically distinct. Also, if sampling intervening tissue reveals continuity; invasive carcinoma associated with an IPMN is favored over concomitant. 5)Lesions resembling genetically engineered rodents acinar-to-ductal metaplasia/atypical flat lesions exist in the human pancreas and may be an alternate pathway of cancer formation. However, their biological significance requires further study.

Conclusions: These revised terminology and recommendations are expected to help improve practice in management of pancreatic precursor lesions, and our understanding of these processes.

\section{Molecular Characteristics of the Pancreatic Intraductal Tubulopapillary Neoplasm}

Umesh Bhanot, Olca Basturk, Michael Berger, Ronak Shah, Sasinya Scott, Volkan Adsay, Gokce Askan, Fatima Carneiro, David Klimstra. Memorial Sloan Kettering Cancer Center, New York, NY; Emory University, Atlanta, GA; IPATIMUP, Porto, Portugal. Background: Intraductal tubulopapillary neoplasm (ITPN)is a relatively recently described member of the pancreatic intraductal neoplasm family. By whole exome sequencing studies, the other member, intraductal papillary mucinous neoplasm (IPMN), was found to have several somatic mutations, often including KRAS, GNAS, and RNF3 genes, in the majority of cases. However, the molecular characteristics of ITPN have not been characterized.

Design: Eleven ITPNs were identified in our institutions' surgical pathology files (10 with matched normal tissue). All neoplasms revealed the characteristic histomorphology - cellular intraductal nodules of back-to-back tubular glands lined by predominantly cuboidal cells with round to oval and atypical nuclei and no obvious intracellular mucin. All tested neoplasms immunolabeled with ductal differentiation markers (CK7, CK19, CA19-9, MUC1) but were negative for intestinal (CK20, CDX2, MUC2), acinar (trypsin, chymotrypsin) and neuroendocrine (chromogranin, synaptophysin) markers. The specimens were microdissected, and deep coverage, targeted next-generation sequencing was performed on a panel of 300 key cancer-associated genes.

Results: The number of mutations per case ranged from 0 to 6 (median=2). None of the previously reported IPMN-related alterations were identified in these ITPNs. In fact, three cases (27\%) did not reveal any mutations in the tested genes and only 2 specific genes were mutated in more than one case: $M L L 2$ and $M L L 3$, each identified in 2 cases. Six cases revealed amplification of $M C L 1$.

Conclusions: Intraductal tubulopapillary neoplasm is a distinct clinicopathologic entity in the pancreas. Although its intraductal nature and some clinicopathologic features resemble those of IPMN, our results suggest that it has distinguishing molecular characteristics. Further analysis of molecular alterations in this biologically distinct pathway including $M L L 2$ and $M L L 3$ (chromatin remodeling genes) will likely shed new light on the mechanisms of intraductal tumor formation in the pancreas and may uncover a new therapeutic target for patients whose tumors harbor these alterations.
1762 Microscopic Invasion in Intraductal Papillary Mucinous Neoplasms of Pancreas

Deyali Chatterjee, Matthew Katz, Asif Rashid, Hua Wang, Gauri Varadhachary, Robert Wolff, Jeffrey Lee, Peter Pisters, Anirban Maitra, Jason Fleming, Huamin Wang. Baylor College of Medicine, Houston, TX; University of Texas MD Anderson Cancer Center, Houston, TX.

Background: Intraductal papillary mucinous neoplasm (IPMN) is a grossly and radiologically distinct cystic neoplasm of the pancreas, $40-50 \%$ of which are associated with a component of invasive carcinoma. The prognosis is excellent for patients with non-invasive IPMN and poor for patients with IPMN with an invasive carcinoma. However the significance of microscopic invasion in IPMN is not clear.

Design: All patients who were diagnosed with IPMN and underwent resection between January 1999 and December 2013 were included. All cases were reviewed for the degree of dysplasia, presence or absence of invasion, lymph node status, total sampling of the tumor, and pancreatic margin status. Microscopic invasion was defined as either unifocal or multifocal invasion of $\leq 5.0 \mathrm{~mm}$ with no grossly identified invasive component. Statistical analyses were performed using SPSS Statistics.

Results: There were a total of 141 IPMN cases ( 54 females and 87 males) with median age of 67 years. IPMN with microinvasion was identified in 8 cases $(5.7 \%)$. The clinical and follow up information was available in 117 patients (31 cases with low grade dysplasia, 29 with high grade dysplasia, 6 with microinvasion, 51 with invasive adenocarcinoma). Among 6 IPMN with microinvasion, 3 were located in the head, 2 in the body, 1 with diffuse involvement of entire pancreas and none had lymph node metastasis. Total sampling of the tumor was performed for 4 cases. During follow up two patients developed recurrence: one with local recurrence at $42 \mathrm{mo}$. who is alive with disease at the last followup, and one with metastasis to lung at $37 \mathrm{mo}$. who died of disease at $68 \mathrm{mo}$. For both cases, the tumors were not complete sampled. None of the 4 cases with complete sampling developed recurrence. Patients with IPMN and microinvasive carcinoma had similar survival to those with non-invasive IMPN and had better survival than those with an IPMN with invasive carcinoma.

Conclusions: Our study showed that patients with IPMN and microinvasion had similar prognosis to those with non-invasive IPMN and had better survival than those with IPMN with invasive carcinoma. Our study highlights the importance of complete tumor sampling in IPMN without grossly identified invasive component.

\section{Histopathologic Features and Clinical Significance of Localized} Peritoneal Invasion By Pancreatic Ductal Carcinoma

Jey-Hsin Chen, Howard Wu, Safi Shahda, Eunice Choi, Zijin Zhao, Jingmei Lin, Elena Chiorean. Swedish Medical Center, Seattle, WA; Indiana University, Indianapolis, IN; University of Washington, Seattle, WA.

Background: Pancreatic ductal carcinoma is a highly malignant neoplasm with a propensity for aggressive locoregional invasion and metastatic spread. The pancreas is partially invested by the peritoneum, which when breached may increase the risk for intra-abdominal dissemination and recurrence. The histopathologic features of peritoneal invasion by pancreatic ductal carcinoma and their clinical significance have not been evaluated to date

Design: 83 surgical pathology cases ( 81 resections and 2 wedge biopsies) of pancreatic ductal carcinoma and variants involving the body and/or tail with extra-pancreatic extension were analyzed using routine and elastic stains and immunostains including pan-keratin and calretinin. The extent of tumor invasion of the peritoneum was divided into 3 groups: 1) no peritoneal involvement, 2) tumor invasion through the elastic lamina and into the submesothelial stromal tissue, and 3) tumor penetration of the peritonea surface. The clinical history and data from institutional cancer registries were reviewed to determine the times and patterns of disease recurrence.

Results: Tumor penetration of the peritoneum involves stepwise invasion of the elastic lamina, subserosa, and mesothelial-lined surface. Invasion of the subserosa is often associated with thinning, fragmentation, and retraction of the elastic lamina from the peritoneal surface and expansion of the submesothelial stroma. Of the 83 cases, 33 tumors did not involve the peritoneum, 20 invaded the elastic lamina and subserosa, and 30 perforated the peritoneal surface. Tumor invasion of the peritoneum (peritoneal surface and/or subserosa) was associated with an increased risk for recurrence (odds ratio $6.75,95 \% \mathrm{CI}=1.84$ to 24.80 ), and all were associated with intra-abdominal recurrence and spread including malignant ascites.

Conclusions: Invasion of the peritoneum is accompanied by architectural alteration that includes fragmentation and retraction of the elastic lamina and expansion of the submesothelial connective tissue. Localized peritoneal invasion by pancreatic carcinoma is associated with a significantly increased risk for tumor recurrence, particularly within the abdominal cavity, which may account for a mechanism for treatment failure in the surgical management of pancreatic carcinoma.

\section{Genomic Characterization of Neoplastic Progression in Pancreatic} Mucinous Cystic Neoplasm

James Conner, Elizabeth Garcia, Adrian Marino-Enriquez, Martha Pitman, Mari MinoKenudson, Lynette Sholl, Amitabh Srivastava, Leona A Doyle. Brigham \& Women's Hospital and Harvard Medical School, Boston, MA; Massachusetts General Hospital and Harvard Medical School, Boston, MA.

Background: Pancreatic ductal adenocarcinoma arises from three morphologically distinct precursor lesions: pancreatic intraepithelial neoplasia (PanIN), intraductal papillary mucinous neoplasm (IPMN), and mucinous cystic neoplasm (MCN). Genetic alterations underlying progression to malignancy have been described for PanIN and IPMN. The aim was to characterize the genomic events underlying progression in MCN, for which more limited data is available.

Design: Microdissected neoplastic epithelium from 24 FFPE specimens was analyzed by massively parallel sequencing using Oncopanel; 15 samples with low-grade dysplasia 
(LG) and 9 with high-grade dysplasia or invasive carcinoma (HG) from 22 patients. Both low and high grade components were evaluated separately in 2 patients. Exonic DNA of 300 cancer associated genes was sequenced, as well as 113 introns across 35 genes to detect rearrangement. Single nucleotide variants (SNVs) and indels were detected using MuTect and GATK. VisCapCancer was used to detect copy number variation. Results: KRAS mutations were most frequent, present in $9(100 \%) \mathrm{HG}$ and $3(20 \%) \mathrm{LG}$ tumors. HG lesions and a LG with concurrent HG showed missense mutations at Gly12 (9) and Gly13 (1), whereas variants in the 2 LG only cases consisted of a Gln61His mutation and an in frame deletion at Lys177. TP53 mutations were identified in $5(56 \%)$ HG cases ( 3 nonsense, 1 frameshift, and 1 splice site mutation) but no LG cases. One $S M A D 4$ mutation was identified in a $\mathrm{HG}$ case $(11 \%)$. Additional genes with mutations in HG but not LG tumor included CTNNB1, PIK3CA, DNMT3A, ERCC4, MLL2, MSH2, STAG2 (in a TP53 mutant case), TCF7L2 (in a CTNNB1 mutant case), and U2AF1. Increased aneuploidy was observed in $\mathrm{HG}$ compared to LG cases, but FGFR3 loss was present in $27 \%$ LG cases vs. $11 \%$ of $\mathrm{HG}$ cases. CDKN2A loss was present in $5(56 \%)$ HG tumors, 3 with codeletion of $C D K N 2 B$, but not in LG tumors. Low copy number gain of GATA6 was detected in 4 (44\%) HG cases and 4 (27\%) LG cases.

Conclusions: Although KRAS mutations are not specific for HG neoplasia, different mutations are detected in those with only LG neoplasia. TP53 mutations and $C D K N 2 A / B$ deletions are common in $\mathrm{HG}$ MCNs and are not seen in LG neoplasms suggesting that these mutations may be used as diagnostic markers preoperatively to identify lesions that warrant resection. Novel SNVs that occur at a lower rate demonstrate molecular heterogeneity in progression pathways and may provide a substrate for new diagnostic, prognostic and therapeutic targets.

1765 Tissue Transglutaminase II Is a Novel Biomarker for Pancreatic Ductal Adenocarcinoma on Core Needle Biopsy and Resection Specimens Todd DeJulio, Xiaoqi Lin, Haonan Li, Xiaoming You, Yihe Yang, Jie Liao, Nike Beaubier Sambasiva Rao, Guang-Yu Yang. Northwestern University, Chicago, IL.

Background: Pancreatic core needle biopsies are often difficult because of scant cellularity, a superficial biopsy or well-differentiated appearance. Many diagnostic features of ductal adenocarcinoma present on resections, such as perineural invasion, haphazard pattern, desmoplasia and anisonucleosis, are absent on core biopsies. Thus, identifying novel biomarkers for ductal adenocarcinoma would be a valuable tool in more accurately and definitively diagnosing these tumors. Multiple global gene expression modalities have shown overexpression of tissue transglutaminase II (TGM-2) in ductal adenocarcinoma, while TGM-2 is minimally expressed in nonneoplastic pancreatic ductal epithelium. In this study, TGM-2 expression is evaluated immunohistochemically on ductal adenocarcinoma and benign pancreatic core needle biopsies and on major resection specimens.

Design: Twenty-five ductal adenocarcinoma and six benign pancreatic core biopsies an sixty nine resected pancreatic ductal adenocarcinomas were assessed. Paraffin sections were labeled with a rabbit monoclonal antibody to TGM- 2 with appropriate positive and negative controls. Cases were scored as positive if dense cytoplasmic labeling was observed. Internal vascular endothelial cells served as a positive control.

Results: More than $50 \%$ of the core biopsies were superficial in nature, consisting only of fragments of atypical epithelium without supporting stroma or acinar tissue. TGM-2 IHC showed that $80 \%(20 / 25)$ of pancreatic ductal carcinomas on core needle biopsy and $78 \%(54 / 69)$ on resected specimens showed diffuse and strong positive staining for TGM-2. All six of the benign pancreatic core needle biopsy cases and all adjacent normal pancreatic parenchyma in the resected specimens showed no positive staining for TGM-2. Capillary endothelial cells and desmoplastic stroma adjacent to invasive carcinoma in the resected specimens displayed intense TGM-2 staining.

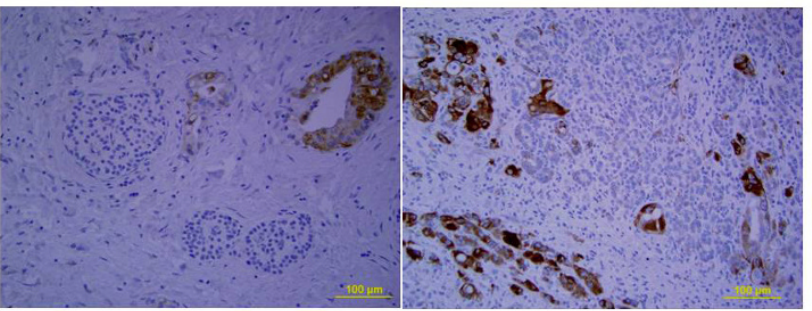

Fig.1. TGM-II staining in pancreatic ductal adenocarcinoma on core needle biopsy

Conclusions: Our results indicate that TGM-2 is a novel biomarker for pancreatic ductal adenocarcinoma that is sensitive and specific and is useful in supporting the diagnosis on core needle biopsies. Overexpression of TGM-2 in cancer-adjacent endothelial cells and desmoplastic stroma imply its role in tumor-stromal interaction and angiogenesis.

1766 Expression of TTMP - A Novel Growth Inhibition Gene Is Lost in Pancreatic Neuroendocrine Tumor

Xianzhong Ding, Hongfa Zhu, Jinping Lai, Lijun Huang, Mona Cornwell, Susan Crawford. Loyola University Medical Center, Maywood, IL; Mount Sinai Medical Center, New York, NY; Saint Louis University School of Medicine, St. Louis, MO.

Background: Using a cDNA microarray technique, we have identified a novel gene that is markedly induced by phorbol-12-myristate-13-acetate (TPA). Using Rapid Amplification of cDNA Ends technology, we have successfully cloned the 2254 base-pair full-length cDNA of this gene. The predicted open reading frame is from nucleotide 20 to nucleotide 673 of the cDNA and codes for a protein with 217 amino acid residues. Computational analysis predicted that the encoded product of this gene is a type II trans-membrane protein sited across the endoplasmic reticulum (ER) membrane. Therefore we named this gene product TPA-induced Transmembrane Protein (TTMP)
A polyclonal antibody has been successfully generated from rabbits immunized with conjugated synthetic peptide. The current project is to study the expression pattern of TTMP in human pancreatic islets and pancreatic neuroendocrine tumors and to explore its role in regulating cell proliferation.

Design: Immunostain for TTMP was performed on 19 well differentiated pancreatic neuroendocrine tumors with paired non-neoplastic pancreas. Based on intensity, the immunostain was graded as no staining $(0)$, low intensity staining $(+)$ or high intensity staining $(++)$. To explore the functional effect of TTMP on cell proliferation, overexpression of TTMP was introduced by transfecting TTMP cDNA. Effect of TTMP on cell proliferation was evaluated by cell count in vitro. Statistical analysis was performed by using Fisher's exact test and student's t test.

Results: Immunostains show a strong TTMP expression (2+) in normal pancreatic islet cells with an intracytoplasmic granular staining pattern. In contrast, there is a complete loss of TTMP expression in all of the well differentiated pancreatic neuroendocrine tumors tested $(100 \% ; 19 / 19)$. Pancreatic acinar and ductal cells are positive for TTMP but with only very weak cytoplasmic staining $(0$ to $1+)$. In vitro study shows TTMP overexpression significantly inhibits human cell proliferation $(\mathrm{p}<0.01)$.

Conclusions: Expression of TTMP is completely lost in well differentiated pancreatic neuroendocrine tumors while TTMP overexpression blocks cell proliferation. Our results suggest that TTMP might be involved in pancreatic neuroendocrine tumorigenesis and progression. TTMP could be a useful marker in distinguishing pancreatic neuroendocrine tumor from normal pancreatic islets.

1767 Next-Generation Sequencing and Fluorescence In Situ Hybridization in the Analysis of Bile Duct Brushings for Malignancy Jonathan Dudley, Zongli Zheng, Long Le, Julie Batten, Kathy Vernovsky, Brenda Sweeney, Ronald Arpin, William Brugge, Martha Pitman, Anthony Iafrate. Massachusetts General Hospital, Boston, MA.

Background: Bile duct brushing of pancreatic and biliary ducts is a specific but insensitive test for the assessment of malignancy. This study assesses the benefit of molecular testing with next-generation sequencing (NGS) relative to fluorescence in situ hybridization (FISH) in the accurate detection of malignancy.

Design: Bile duct brushings collected from nineteen patients were subjected to cytological analysis, FISH using the UroVysion probe set, and targeted NGS using the $\mathrm{SNaPshot}$ assay. Specimens were placed into "negative"/"atypical" [true negative] or "suspicious"/"positive" [true positive] categories based on cytology and "negative" or "positive" categories based on FISH and NGS results. Performance characteristics for each diagnostic modality were calculated based on clinical follow-up or subsequent pathology indicating malignancy.

Results: There were 10 malignant strictures (53\%) and 9 benign based on radiologic and/or follow-up pathological data. NGS revealed driver mutations in KRAS, p53, and or SMAD4 in 7/19 (37\%) cases, including 6 of 9 patients with malignancy $(67 \%)$. One KRAS mutant sample was benign on subsequent pathology (cholecystitis), possibly reflecting an unresected precursor lesion (e.g. biliary intraepithelial neoplasia). NGS for 2 patients failed. FISH results were positive in 7/19 (37\%) samples, including 6 of the $10(60 \%)$ patients with malignancy. Cytology was positive in 7 patients $(37 \%)$, suspicious in 2, atypical in 2, and negative in 8 . Cytology was positive or suspicious in 9 of the 10 patients with malignancy. Table 1 summarizes the performance characteristics of each modality.

\begin{tabular}{|c||c||c||c||c||c||}
\hline & NGS & FISH & Cytology & Cytology+NGS & Cytology+FISH \\
\hline \hline Sensitivity (\%) & 67 & 60 & 90 & 100 & 90 \\
\hline \hline Specificity (\%) & 88 & 89 & 100 & 89 & 89 \\
\hline \hline Accuracy (\%) & 76 & 74 & 95 & 95 & 89 \\
\hline
\end{tabular}

Conclusions: NGS and FISH have similar performance characteristics in the analysis of bile duct brushing specimens, with NGS increasing the sensitivity in the detection of malignancy to $100 \%$. When adjunctive molecular testing is sought, NGS may be preferred over FISH in light of its higher accuracy with comparable specificity, increased sensitivity, and significantly decreased cost and complexity.

1768 Adenomyomatous Nodules of the Gallbladder: Clinicopathologic Analysis of 234 Cases, Including 20 With Neoplastic Transformation Nevra Dursun, Oguzhan Okcu, Bahar Memis, Gizem Akkas, Rhonda Everett, Olca Basturk, Pelin Bagci, Serdar Balci, Burcu Saka, Brian Quigley, A Krasinskas, Juan Roa, Kee-Taek Jang, Juan Sarmiento, Volkan Adsay. Emory University, Atlanta, GA; Wayne State University, Detroit, MI; Pontificia Universidad Católica, Santiago, Chile; Sungkyunkwan University, Seoul, Korea.

Background: The nature and clinicopathologic associations of "adenomyoma" or "adenomyomatous hyperplasia" (AM) of gallbladder (GB) are poorly characterized. A recent study (PMID:22038508) claimed AM as the source of malignancy in $26 \%$ of GB carcinoma.

Design: Through systematic review and targeted search, 234 AMs were identified and analyzed.

Results: Clinical: Prospectively reviewed 1000 consecutive GBs revealed AM in $6.8 \%$. Mean age $=58$ (vs 50 in the entire cohort). $\mathrm{F} / \mathrm{M}=1.8$ (vs 3.4). Mean size=1.3 $\mathrm{cm}(0.5-5.9)$. Pathology: Most (98\%) were in the fundus; 4 were multifocal; 3 were diffuse ("adenomyomatosis"). AM presented as well-demarcated, nodular area of wall thickening, often forming a dimple in the overlying mucosa. Microscopically, a conglomeration of dilated glands (up to $14 \mathrm{~mm}$ ), often radially converging to a point in the mucosa, but not individually opening to the surface (in contrast to RokitanskyAschoff sinuses) was noted. Variable amounts of muscle, often minimal around the deeper glands/cysts, were present. 9 (4\%) revealed overt features of a duplication, by 
connecting to a well-defined lumen with circumferential muscle layer. Associations: Pyloric gland metaplasia was less common than controls ( $38 \%$ vs $52 \%$ ); no other specific associations with stones, inflammation, cholesterolosis, intestinal metaplasia or thickening of the uninvolved GB wall (mean=3.6 $\mathrm{mm}$ vs $3.3 \mathrm{~mm}$ in control) were identified. Neoplastic change: There was prominent mucinous change in the epithelium in $23(10 \%)$ AMs; 16/23 with papilla formation ("mural IPMN" pattern). 7 (3\%) AMs had high-grade dysplasia/CIS. 13 cases had both AM and invasive carcinoma: In 2, $\mathrm{AM}$ and carcinoma were separate; in 6, AM appeared to be secondarily involved by carcinoma (innocent bystander). In only $5(2 \%)$, carcinoma was arising from AM (invasion was confined to AM area, measuring 0.1-0.9 cm, and dysplasia was either confined to AM area or predominantly in AM).

Conclusions: $\mathrm{AM}$ is seen in $<7 \%$ of cholecystectomies. It does not have association with GB injury or evidence of being neoplastic itself, but rather appears to be a developmental/malformative process, possibly a version of a duplication/diverticulum. Thus, adenomyomatous nodule may be a more accurate name. Mucinous change with papillary units ("mural IPMN" pattern) occurs in 7\%. High-grade dysplasia is seen in $3 \%$ and invasive carcinoma, often small, arises in $2 \%$.

\section{Immunohistochemistry for IgE Can Distinguish IgG4-Related} Disease of the Pancreatobiliary Tract From Chronic Pancreatitis

Michael Feely, Lizette Vila Duckworth, David Gonzalo. University of Florida, Gainesville, FL.

Background: Although IgG4-related disease (IRD) of the pancreas and biliary tract is a true clinicopathologic diagnosis, the recognition of this entity relies on the pathologist's ability to identify key histologic findings including lymphoplasmacytic infiltrates, storiform fibrosis and obliterative phlebitis. In addition, quantification of IgG4 and IgG positive plasma cells by immunohistochemistry is a major criterion, an interpretation of which can prove difficult at times and lead to inconclusive results. A recent study investigating cytokine production in IRD of the salivary gland noted the presence of mast cells with strong cytoplasmic IgE immunoreactivity. Based upon these findings, this study aimed to assess the presence of mast cells and IgE positive cells in IRD of the pancreas and biliary tract and to evaluate their utility in the diagnosis of this entity. Design: Twelve cases of IRD of the pancreas and biliary tract were retrieved from the pathology archives along with five cases each of normal pancreas and chronic pancreatitis. Immunohistochemistry for IgE and c-Kit were performed on a representative block from each case. The number of cells displaying strong cytoplasmic immunoreactivity for IgE as well as c-Kit positive mast cells were quantified and compared among the individual diagnostic categories.

Results: Cases of IRD contained a mean number of 1.75 cells with strong IgE cytoplasmic staining per high power field (HPF) in areas of highest concentration. In stark contrast, no case of chronic pancreatitis or normal pancreas contained cells with strong cytoplasmic IgE immunoreactivity. The presence of cells strongly positive for $\operatorname{IgE}$ was $91.6 \%$ sensitive and $100 \%$ specific for IRD. While there was a statistical difference in the mean number of mast cells in IRD cases (43.6/HPF) versus normal pancreas $(14.2 / \mathrm{HPF})(\mathrm{p}=0.007)$, there was no significant difference in the number of mast cells in IRD cases compared to chronic pancreatitis $(50.6 / \mathrm{HPF})(\mathrm{p}=0.294)$.

Conclusions: Our study shows that strong cytoplasmic staining for $\operatorname{IgE}$ is a highly specific and sensitive marker for IRD. This finding can be very useful in the differential diagnosis with chronic pancreatitis and normal pancreas, particularly when assessment of IgG and IgG4 positive plasma cells is uncertain due to inconclusive IgG4/IgG counts or when limited biopsy material is available. The finding of an increased number of mast cells in IRD compared to normal pancreas suggests these cells may have a role in the pathogenesis of this disease.

1770 Prognostic Significance of the Number and Ratio of Positive Lymph Nodes for Patients With Pancreatic Ductal Adenocarcinoma Who Completed Neoadjuvant Therapy and Pancreaticoduodenectomy

Laurice Fischer, Matthew Katz, Sun Mi Lee, Li Liu, Manonmani Sundar, Hua Wang, Gauri Varadhachary, Robert Wolff, Jeffrey Lee, Peter Pisters, Anirban Maitra, Jason Fleming, Jeannelyn Estrella, Asif Rashid, Huamin Wang. University of Texas MD Anderson Cancer Center, Houston, TX.

Background: Lymph node metastasis is a significant prognostic factor in patients with pancreatic ductal adenocarcinoma (PDC). Although the number of positive lymph nodes is currently used to subclassify the $\mathrm{pN}$ stage in carcinomas of other organs, the prognostic significance of the number and ratio of positive lymph nodes in post-therapy pancreaticoduodenectomy (PD) is not clear.

Design: The study population was comprised of 411 patients with PDC who completed neoadjuvant therapy and underwent PD at our institution between 1999 and 2012 (183 women and 228 men, with a median age of 64 years). All PD specimens were processed using a standardized pathologic evaluation system. The lymph node ratio (LNR) was calculated as a ratio of the number of positive lymph nodes to total number of lymph nodes dissected. The number of positive lymph nodes and LNR were correlated with clinicopathologic parameters and survival using SPSS statistics.

Results: The total number of lymph nodes examined ranged from 5 to 68 , with a median of $22.189(46 \%)$ of patients were node negative, while $222(54 \%)$ of patients were node positive. Patients with node-negative disease had better disease-free survival (DFS) and overall survival (OS) than those with node positive disease $(\mathrm{P}<0.001)$. Within the node-positive group, $79(35.6 \%)$ had one positive lymph node, $143(64.4 \%)$ had two or more positive lymph nodes, $166(74.8 \%)$ had LNR $<0.194$ (75th percentile value of LNR) and $56(25.2 \%)$ had LNR $\geq 0.194$. Patients with one positive lymph node had better DFS $(\mathrm{p}=0.002)$ and $\mathrm{OS}(\mathrm{p}=0.001)$ than those with $>1$ positive lymph node. Similarly, patients with LNR $<0.194$ had better DFS $(p<0.001)$ and $\operatorname{OS}(p<0.001)$ than those with LNR $\geq 0.194$. In multivariate analysis, both the number of positive lymph nodes and LNR are independent prognostic factors for DFS and OS.
Conclusions: The number of positive lymph nodes and LNR are independent prognostic factors for survival in patients with PDC who completed neoadjuvant therapy and underwent $\mathrm{PD}$. Subclassification of the $\mathrm{N} 1$ group to $\mathrm{N} 1 \mathrm{a}$ (one positive lymph node) and $\mathrm{N} 1 \mathrm{~b}(>1$ positive lymph node) is justified within this group of patients.

1771 Multifocal Pancreatic Neuroendocrine Tumors: Clonality and miRNA Profiling

Arunima Ghosh, Ang Li, Gregory Brown, Electron Kebebew, Maria Merino. National Cancer Institute, Rockville, MD.

Background: Pancreatic neuroendocrine neoplasms (PNNs) occur sporadically and in patients with multiple endocrine neoplasia type 1 (MEN1). In MEN1, multiple tumors have been shown to arise by independent clonal events. The purpose of the present study was to analyze clonality and microRNA (miRNA) profiling in neuroendocrine tumors from four MEN1 patients in whom the tumor was present in at least two separate sites, which included the pancreas, duodenum and the retroduodenal or peri-pancreatic lymph nodes. In one patient, there was tumor involvement in the right and left ovaries. Design: Clonality was assessed by MEN1 gene mutation and human androgen receptor analysis(HUMARA) assays. Normal and tumor DNA was purified from microdissected tissue by Qiagen DNA isolation kit. For the HUMARA assay, DNA was digested with HpaII or no enzyme; products were subjected to PCR with HUMARA primers and analyzed on the ABI 310 genetic analyzer. Total RNA was extracted using RecoverAll ${ }^{\mathrm{TM}}$ Total Nucleic Acid Isolation Kit (Ambion, Austin, TX, USA). MiRNAs expression was profiled using nCounter Human v2 miRNA Expression Assay (NanoString Technologies, Seattle, WA, USA) and raw data was analyzed using nSolver Analysis Software (V.1.1) and Excel. Background threshold was defined as the mean $\pm 1 \mathrm{SD}$ of negative controls. The expression difference was indicated as mean fold change.

Results: All the patients were positive for MEN1 gene mutation. HUMARA analysis in two of four patients revealed identical clonal patterns in the tumors from multiple sites in each patient. The clonal composition of the PNNs was not associated with a particular growth pattern, proliferation index or immunohistochemical expression pattern. Of the other two, one site was monoclonal which may represent an initial seeding event. The profiling of 800 miRNAs showed differential miRNA expression between tumor tissues from different sites; 32 miRNAs showed increased expressions (miR-193b-3p, miR-215, miR-196b-5 notably) while 16 miRNAs were down-regulated (miR-148a-3p, miR-204-5p, miR-375). Interestingly, miR-210 was also upregulated in these samples, and this miRNA has been implicated in metastatic disease.

Conclusions: These findings suggest that PNNs might initially represent poly/ oligoclonal neoplastic lesions which are eventually outgrown by a single, more aggressive cell clone with the potential for invasive growth and metastatic spread. The differentially expressed miRNAs may provide insights into the understanding of this cancer, and provide new tools for the development of new molecular targets.

\section{MicroRNA Associated With Outcome in Pancreatic Neuroendocrine} Tumors

Jose Gonzalez-Berjon, Michael Tetzlaff, James Yao, David Loose, Russell Broaddus, Jeannelyn Estrella. University of Texas MD Anderson Cancer Center, Houston, TX; University of Texas Health Medical School, Houston, TX; Health Sciences North|Horizon Santé-Nord, Sudbury, ON, Canada.

Background: Pancreatic neuroendocrine tumors (PanNETs) have a variable clinical course, ranging from indolent to highly aggressive. Current clinicopathologic parameters variably predict outcome, but there is still considerable outcome heterogeneity even among patients with the same tumor grade/stage. There is therefore a critical need to define additional biomarkers that more reliably stratify risk. MicroRNA (miRNA) expression has been shown to play an important prognostic role in other cancer types. Here, we demonstrate differential miRNA expression among patients with stage matched PanNET, but with different clinical outcomes.

Design: We evaluated 45 PanNETs: (a) 20 stage IV [10 died of disease $<7$ y (wors survivors) +10 with $>7$ y survival (best survivors) ] and (b) 25 stage I-IIB [18 developed distant metastasis $<7 \mathrm{y}$ (metastasis) +7 without metastasis $>7 \mathrm{y}$ follow-up (no metastasis)]. miRNA expression profiling was performed using PCR-based analysis (Exiqon miRNAome). Difference of means $(\mathrm{dm})=$ difference between group 1 and group 2 mean $C_{t}\left(\Delta \Delta C_{t}\right)$ in log scale. Fold change $(F c)=2^{\Delta \Delta C t}$. Student's t-test was used to compare between groups.

Results: 9 miRNAs were differentially expressed between stage IV worst and best survivors while 40 miRNAs were differentially expressed between stage I-IIB metastasis and no metastasis groups. Selected microRNAs are summarized in Table 1 (based on $P, \mathrm{dm}$ and fc values).

\begin{tabular}{|c|c|c|c|}
\hline $\begin{array}{l}\text { Worst survivors }(\mathrm{n}=10) \text { versus Best survivors } \\
(\mathrm{n}=10)\end{array}$ & $\begin{array}{c}\text { Difference of means } \\
(\Delta \Delta \mathrm{Ct})\end{array}$ & $\begin{array}{l}\text { Fold } \\
\text { Change }\end{array}$ & $P$ value \\
\hline hsa-miR-432-3p & -1.71 & -3.26 & 0.016 \\
\hline hsa-miR-206 & -0.95 & -1.94 & 0.033 \\
\hline hsa-miR-212-3p & 0.95 & 1.93 & 0.018 \\
\hline hsa-miR-15a-3p & 0.76 & 1.70 & 0.025 \\
\hline \multicolumn{4}{|l|}{ Metastasis $(n=18)$ versus No metastasis $(n=7)$} \\
\hline hsa-miR-144-3p & -2.06 & -4.17 & 0.014 \\
\hline hsa-miR-129-2-3p & -1.78 & -3.43 & 0.019 \\
\hline hsa-miR-455-5p & 1.93 & 3.81 & 0.018 \\
\hline hsa-miR-1179 & 1.55 & 2.93 & 0.019 \\
\hline
\end{tabular}


Conclusions: Differentially expressed miRNAs correlate with distinctive patient outcomes among PanNETs and may represent important surrogates of risk. Validation in a large, independent cohort of patients is needed to construct a miRNA signature predictive of outcome in these heterogeneous tumors.

1773 Follicular Pancreatitis: A Mimic of Pancreatic Malignancy

Rajib Gupta, Vikram Deshpande, Kurt Patton, Stephen Behrman, Bill Xie. University of Tennessee Health Science Center, Memphis, TN; Massachusetts General Hospital and Harvard Medical School, Boston, MA; Pathology Group of Midsouth, Germantown, TN. Background: Follicular pancreatitis is a recently described variant of chronic pancreatitis characterized by the formation of a discrete pancreatic mass and histologically by the presence of multiple reactive lymphoid follicles and the absence of histo-serologic features of autoimmune pancreatitis. This rare entity, recently reported from Asia in 2012, has not been reported in North America.

Design: All pancreatic resections with a diagnosis of chronic pancreatitis were reevaluated. The histopathologic diagnosis of follicular pancreatitis was based on the presence of a prominent infiltrate of reactive germinal centers. Relevant clinical, radiologic and demographic data were collected. Immunohistochemistry for IgG4, Bcl2 and B- and T-cell markers were performed on the pancreatic tissue.

Results: We identified 3 cases from our institutions which conformed to the histopathologic diagnosis of follicular pancreatitis. Two were pancreatectomy specimens and one was a core biopsy. The mean age of the patients was 57 years with a slight male predominance ( $\mathrm{M}: \mathrm{F}=2: 1$ ). Pancreatic tumor was diagnosed incidentally in 2 cases while one patient presented with epigastric pain and fatigue. One case reported a history of ulcerative colitis. Radiologically, 2 patients showed a mass in the tail and body respectively while one (the symptomatic patient) showed only a diffuse dilatation of the pancreatic duct. Serum IgG4 was not elevated in two of the three cases examined. Histopathologic examination in all three cases showed numerous reactive germinal centers, located predominantly around pancreatic ducts. Storiform type fibrosis was not observed. A focus of obliterative phlebitis was seen in one case. Neutrophils were not present. The mean IgG4+ plasma cells was 10 per high power field and IgG4 to IgG ratio $<30 \%$. The germinal centers were negative for $\mathrm{Bcl} 2$ and there was no evidence of a monoclonal B-cell proliferation.

Conclusions: Follicular pancreatitis is a distinct variant of chronic pancreatitis that clinically and radiologically may mimic a pancreatic neoplasm.

1774 High Throughput Mutational Profiling of Biliary Neuroendocrine Carcinomas

Soon Auck Hong, Sung-Min Chun, Hosub Park, Soyeon An, Kyu-pyo Kim, Eunsil Yu, Se-Jin Jang, Seung-Mo Hong. Asan Medical Center, University of Ulsan College of Medicine, Seoul, Korea.

Background: Biliary neuroendocrine carcinomas (NECs) are extremely rare with worse prognosis. Due to its rarity, no well-established therapeutic modality have been set up for treatment of biliary NECs. Comparing with neuroendocrine tumors from other organs, one of the characteristics of biliary NECs are mixed adenoendocrine carcinomas (MANECs) which consist of mixed neuroendocrine carcinomas with conventional cholangiocarcinomas. To the best of our knowledge, somatic mutational patterns of biliary NECs have not been well elucidated.

Design: DNA was extracted from FFPE tissues from 14 (7 NECs and 5 MANECs) biliary NECs, and followed by preparation of library for targeted NGS with OncoPanel Version 2 probe set focused on cancer related 505 genes. Captured sequences were amplified then allowed to Massive Parallel Sequencing using MiSeq platform.

Results: NEC component in pure NEC were classified as 6 small cell carcinomas and 1 large cell carcinoma. In MANECs, all 5 were small cell carcinomas. Commonly mutated genes included MUC2 (9/12, 75\%), TP53 (8/12, 67\%), RB (8/12, 67\%), HLA-A (5/12, $42 \%), H L A-D R B 1$ (4/12, 33\%), NOTCH2 (4/12, 33\%), CREBBP (3/12, 25\%), AFF (3/12, 25\%). Other mutations, including ERBB3, KATA6, LAMA5, KMT2D, NOTCH4, SYNE1 ( 2 cases each, 17\%), APC, GNAS, and MYCL (1 case each, $8 \%$ ) were detected at lower frequencies.

Conclusions: Mutational profiling of biliary NECs demonstrate combined mutational patterns of small cell carcinomas and cholangiocarcinomas. Understanding these unique mutational pattern may be helpful for proper treatment of biliary NEC patients.

1775 Serous Neoplasms of Pancreas: Analysis of 231 Cases With Emphasis on Variants and Reappraisal of "Serous Cystadenocarcinoma" Choi Hyejeong, Bahar Memis, Olca Basturk, Gizem Akkas, Kee-Taek Jang, Volkan Adsay, Michelle Reid. Emory University Hospital, Atlanta, GA; Memorial Sloan Kettering Cancer Center, New York, NY; Samsung Medical Center, Seoul, Korea. Background: The literature on variants and "malignant" counterpart of pancreatic serous neoplasms ( $\mathrm{SNs}$ ) remains conflicted.

Design: The clinicopathologic features of 231 SNs were analyzed.
Results:

\begin{tabular}{|l||c||c||c||c||c||c||}
\hline Variant & $\%$ & $\begin{array}{c}\text { Mean } \\
\text { Age }\end{array}$ & F:M & $\begin{array}{c}\text { Mean } \\
\text { Size } \\
(\mathrm{mm})\end{array}$ & $\begin{array}{c}\text { Body/ } \\
\text { Tail vs } \\
\text { Head }\end{array}$ & $\begin{array}{c}\text { Misdiagnosed on Imaging } \\
(\%)\end{array}$ \\
\hline \hline Microcystic & 77 & 61 & 3 & 44 & 1.7 & $\begin{array}{c}\text { Carcinoma (7)NET (7)MCN } \\
\text { (5)IPMN (5)Other (6) }\end{array}$ \\
\hline \hline -Ordinary & 70 & 61 & 3 & 45 & 2 & \\
\hline \hline $\begin{array}{l}\text {-Mixed } \\
\text { micro/ } \\
\text { macrocystic }\end{array}$ & 7 & 57 & 12 & 35 & 0.4 & \\
\hline \hline Oligocystic & 21 & 50 & 4 & 38 & 1.7 & MCN (23)IPMN (8) \\
\hline \hline -Unilocular & 9 & 48 & 4 & 34 & 2.5 & \\
\hline \hline -Multilocular & 12 & 52 & 5 & 41 & 1.3 & \\
\hline \hline Solid & 2 & 58 & 1 & 40 & 1 & NET (75) \\
\hline \hline Total & 100 & 58 & 3 & 41 & 1.7 & \\
\hline \hline
\end{tabular}

Coexisting tumors were not uncommon( $13 \%$ ):NETs, $7 \%$, pancreatic/ampullary ca, $2 \%$ and others, $4 \%$. FNA (was available in $4 \%$ ) and was diagnostic in 1 case. Oligocystic $\mathrm{SNs}(\mathrm{OV})$, previously reported in major texts (WHO/AFIP) as accounting for $40 \%$ of SNs arising in the pancreatic head/neck of older men, constituted only $21 \%$ of our cases, was $1.7 \mathrm{X}$ more common in body/tail and affected younger females (F:M=4); 55\% of OVs were multilocular, $45 \%$, unilocular and they were often misread as mucinous cysts on imaging. Solid SN was least common ( 5 cases) and misread as NET on imaging. No SNs had malignant cytology or malignant behavior except for 1 previously reported consult case. 1 hepatic SN was VHL-associated, had classical SN morphology and was interpreted as an independent SN and not a metastasis. Six larger SNs (mean 113mm) showed adhesion to/direct penetration of adjacent tissues including LNs $(n=4)$, stomach $(\mathrm{n}=1)$, spleen and colon $(\mathrm{n}=1)$

\begin{tabular}{||l||c||c||c||c||c||c||}
\hline \hline Variant & $\%$ & $\begin{array}{c}\text { Mean } \\
\text { Age }\end{array}$ & F:M & $\begin{array}{c}\text { Mean } \\
\text { Size } \\
(\mathrm{mm})\end{array}$ & $\begin{array}{c}\text { Body/ } \\
\text { Tail vs } \\
\text { Head }\end{array}$ & $\begin{array}{c}\text { Misdiagnosed } \\
\text { on Imaging } \\
(\%)\end{array}$ \\
\hline $\begin{array}{l}\text { Adherent/Directly penetrating } \\
\text { adjacent organs/LNs }\end{array}$ & 2.6 & 63 & $4: 2$ & 113 & 2 & $\begin{array}{c}\text { Solid mass } \\
(16) \text { GIST (16) }\end{array}$ \\
\hline
\end{tabular}

Conclusions: Contrary to reported literature, in this the largest series of SNs, the clinicopathologic features of oligocystic and solid variants were virtually identical to those of ordinary microcystic variant. Although larger SNs occasionally $(2.6 \%)$ showed direct adhesion/penetration of neighboring organs including LNs, and occasionally recurred $(<1 \%)$, they did not have malignant cytology or malignant behavior nor were there attributable deaths. True cytologically malignant SNs are extremely rare. Critical appraisal of the literature on malignant SNs reveals similar findings.

1776 Smooth Muscle Actin Expression of Myofibroblasts in Pancreatic Cancer Correlates With Changes in Immune Infiltrate and Tumor-Induced Hypoxia Following Neoadjuvant Therapy

Florencia Jalikis, Yongjian Jiang, Matthew M Yeh, Venu Pillarisetty. University of Washington, Seattle, WA.

Background: Elucidation of the cancer-stroma interaction may hold the key for treating pancreatic ductal adenocarcinoma (PDA), one of the most rapidly lethal human cancers. Previous studies have demonstrated that the tumor microenvironment, composed of immune cells, myofibroblasts and extracellular matrix, plays a role in cancer development, progression and response to treatment. In addition, hypoxia, a defining feature of PDA, contributes to tumor progression. Successful multimodal neoadjuvant therapy have shown to alter the immunoregulatory balance of the tumor microenvironment. We aimed to determine the correlation between myofibroblast density, immune cell infiltrate and tumor-induced hypoxia in PDA following neoadjuvant therapy.

Design: We performed immunohistochemistry (IHC) for smooth muscle actin (SMA) and hypoxia-inducible factor 1-alpha (HIF-1a) in a set of primary PDA upon which we have previously done an extensive characterization of their immune infiltrate. Samples included both, untreated $(\mathrm{n}=18)$ and neoadjuvant-treated cancers $(\mathrm{n}=20)$. Results were interpreted using a visual score and computer-assisted image analysis.

Results: Stromal SMA expression in PDA was moderate $(n=13)$ to high $(n=14)$ in the majority of the 38 cases. Quantification of SMA expression using image capture and analysis software strongly correlated with visual SMA scoring $(r=0.83, p<0.0001)$. A direct relationship between nuclear HIF-1 a expression by carcinoma cells and SMA expression by stromal myofibroblasts was noted. In addition, there was a tight correlation between SMA and CD3 $(r=0.64, p=0.003), F O X P 3+$ regulatory $\mathrm{T}$ cells $(\mathrm{r}=0.62$, $\mathrm{p}=0.004)$, and $\mathrm{CD} 11 \mathrm{~b}(\mathrm{r}=0.55, \mathrm{p}=0.01)$ in neoadjuvant treated tumors.

Conclusions: Expression of SMA in stromal myofibroblasts of PDA following neoadjuvant therapy correlates with hypoxia and induction of a broad immune infiltrate including large numbers of potentially immunosuppressive FOXP3+ regulatory T cells and myeloid cells. 
1777 Impact of Next-Generation Sequencing on the Clinical Impression of Pancreatic Cysts

Martin Jones, Zongli Zheng, Jessica Wang, Emily Albanese, Abdurrahman Kadayifci, Dora Dias-Santagata, Long Le, William Brugge, Carlos Fernandez-del Castillo, Mari Mino-Kenudson, A John Iafrate, Martha Pitman. Massachusetts General Hospital, Boston, MA.

Background: The value of next-generation sequencing (NGS) of pancreatic cyst fluid (PCF) relative to the clinical impression and other pre-operative tests has not been well studied.

Design: Ninety-two PCF specimens from 86 patients were analyzed by cytology, CEA and targeted NGS using the SNaPshot NGS assay. Cysts were classified by radiology and EUS reports (Clinical Impression pre-PCF Analysis) as non-neoplastic, serous cystadenoma (SCA), cystic neuroendocrine tumor (cNET), IPMN, MCN, malignant, or not-specified (NS). NGS results were compared to CEA (cut-off value of $192 \mathrm{ng} / \mathrm{ml}$ ) and to cytology with high-grade atypia (HGA) indicating a high-risk cyst. KRAS or GNAS mutations supported a mucinous cyst and GNAS mutation supported an IPMN Results: Results are summarized in Figure 1. NGS supported the clinical impression in $78 \%$ of cysts. For patients with a NS clinical impression, NGS supported a neoplasm in $29 \%$. IPMN was confirmed by GNAS mutations in $22 / 92$ (24\%). A KRAS mutation reclassified as mucinous $19 \%$ of clinically non-neoplastic cysts with a nonmucinous CEA. One PCF with a clinical impression of cNET had cytology diagnostic of carcinoma. Seven (8\%) PCF had either a TP53 mutation, or loss of CDKN2A or SMAD4 All 7 PCF also had either KRAS and/or $G N A S$ mutations; 5/7 (71\%) were clinically malignant and HGA was detected in $5 / 7(71 \%)$. Overall, cysts nonmucinous by CEA were reclassified as mucinous in $45 \%$ of patients. All PCF with HGA on cytology $(8 / 8)$ had mutations indicating a neoplasm, 6 malignant, 4 confirmed by unresectable imaging and 2 by histology. Histology in 13 patients $(15 \%)$ showed 4 non-neoplastic (1 KRAS), 6 IPMN (all KRAS and 3 GNAS) and 3 malignant cysts (one KRAS, GNAS, TP53; 2 no mutations).

Figure 1.

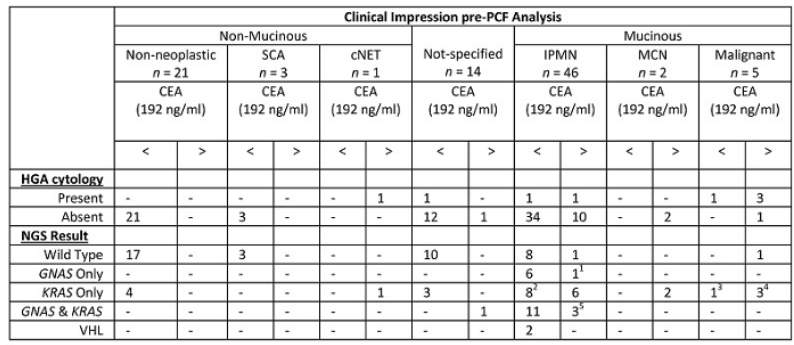

${ }_{5}^{1}+$ loss of COKN2A; ${ }^{2}$ loss of CDKN2A in $1 ;{ }^{3}+$ loss of CDKN2A; ${ }^{4}+$ loss of CDKN2A in 2 and loss of both SMAD4 and CDKN2A in 1 ;

Conclusions: NGS of PCF impacts patient management by defining or supporting a neoplastic process requiring follow-up or resection. In our series it was most valuable in identifying mucinous cysts with CEA $<192 \mathrm{ng} / \mathrm{ml}$. NGS may also offer prognostic information by identifying specific mutations, but this will require further investigation.

1778 Somatostatin Receptor $2 A$ and 5 Expression in Poorly Differentiated Neuroendocrine Neoplasms of Pancreatic and Extrapancreatic Origin

Bjorn Konukiewitz, Anna Melissa Schlitter, Abbas Agaimy, Irene Esposito, Gunter Kloppel. Technische Universität München, Munich, Germany; Universität Erlangen, Erlangen, Germany.

Background: Most well differentiated neuroendocrine neoplasms (NENs) express somatostatin receptors (SSTR), especially SSTR2A and SSTR5. The expression of these SSTRs is of clinical importance for diagnosis, therapy and follow-up of well differentiated NENs. Little is known about the expression of SSTR2A and SSTR5 in poorly differentiated NENs (PDNENs). This study investigates the immunohistochemical SSTR2A and SSTR5 expression in PDNENs of different origin Design: 29 PDNENs (all with a Ki67-index $>20 \%$, including 10 from the pancreas/ ampulla, 7 from the colorectum, 5 from the bladder, 4 from the parotid gland, and 3 Merkel cell carcinomas of the skin) were analyzed by immunostaining for SSTR2A and SSTR5 (using a 3-tiered scoring system), synaptophysin, chromogranin A, CD56, p53, CK 20, and progesterone receptor (PR). Molecular analysis was performed for TP53 mutations. Pancreatic and colorectal PDNENs were compared with well differentiated NENs (WDNENs; with a Ki67-index $>20 \%$ ) of pancreatic $(n=4)$ and colorectal origin $(\mathrm{n}=2)$.

Results: 4/29 PDNENs expressed SSTR2A (score 2 and 3) and 1/29 was SSTR5 positive. The SSTR2A-positive PDNENs resided in the pancreas (1/4), the colorectum (2/4) and the bladder (1/4). 17/25 (68\%) SSTR2A-negative PDNENs showed nuclear p53 expression in more than $5 \%$ (range 7-95\%) of tumor cells. $2 / 4$ of the SSTR2Apositive PDNENs were also $\mathrm{p} 53$ positive. $5 / 6$ WDNENs were negative for $\mathrm{p} 53$ and $4 / 6$ of these tumors were positive for SSTR2A. All pancreatic and extrapancreatic PDNENs were negative for PR. CK20 was mostly detected in colorectal PDNENs and showed strong positivity in all PDNENs of the parotid gland.

Conclusions: SSTR2A expression is not restricted to WDNENs but occurs also in single PDNENs. SSTR2A negativity in PDNENs correlates reciprocally with strong p53 expression. Pancreatic and colorectal WDNENs with a Ki67-index greater than 20\% retain their SSTR2A expression. SSTR5 is only exceptionally expressed in PDNENs.
1779 Comparison of Morphologic Subtypes of Intraductal Papillary Mucinous Neoplasm of the Pancreas With Their Mucin Immunoprofiles

Heewon Kwak, Xiuli Liu, Daniela Allende, Rish Pai, John Hart, Shu-Yuan Xiao. University of Chicago, Chicago, IL; Cleveland Clinic Foundation, Cleveland, OH. Background: Intraductal papillary mucinous neoplasm (IPMN) is divided into four histologic subtypes: gastric (G), intestinal (I), pancreatobiliary (PB), and oncocytic (O). The initial classification primarily used morphology without strict criteria for utilizing immunohistochemistry (IHC). In a concurrent study, we demonstrated moderate interobserver agreement among gastrotintestinal (GI) pathologists in classifying IPMN subtypes by morphology. This study compares IPMN subtypes diagnosed by morphology with their respective mucin (MUC) immunoprofiles.

Design: Fifty IPMN cases were reviewed independently by 5 GI pathologists for IPMN subtyping by $\mathrm{H} \& \mathrm{E}$ morphology. A consensus diagnosis had at least 4 of 5 pathologists in agreement. IHC with MUC1, MUC2, MUC5AC, MUC6, and CDX2 was performed on 40 cases and quantitatively scored: 0 (no staining), $1(<10 \%$ positivity of the lesional cells), 2 (10 to $<50 \%), 3$ (50 to $<75 \%$ ), or $4(>75 \%)$. IHC results were grouped as negative $(0-1)$ or positive (2-4).

Results: IHC was performed on 29 IPMN cases (16 G-types, 8 I-types, 4 PB-types, 1 mixed type) with and 11 cases without a consensus diagnosis. Morphologic diagnosis was concordant with MUC1-, MUC2-, MUC5AC+, and CDX2- for 14/16 G-types and MUC1-, MUC2+, MUC6-, and CDX2+ for 7/8 I-types. MUC6 positivity was seen in 10/16 G-types. PB-types had variable immunoreactivity with all markers except MUC2, which was negative in all cases.

IHC of IPMN Subtypes with a Consensus Diagnosis

\begin{tabular}{|c||c||c||c||c|}
\hline IPMN Subtype (\# of cases) & G (16) & I (8) & PB (4) & Mixed* \\
\hline \hline MUC1+ + & 0 & 0 & 2 & 0 \\
\hline \hline MUC2+ + & 1 & 8 & 0 & 0 \\
\hline \hline MUC5AC+ + MUC6+ & 14 & 6 & 2 & 1 \\
\hline \hline CDX2+ & 10 & 0 & 1 & 0 \\
\hline \hline
\end{tabular}

"Mixed indicates each subtype present comprises $\geq 25 \%$ of thelesion

Eight of 11 cases that lacked a consensus diagnosis had immunoprofiles consistent with either G-types (4 cases with MUC1-, MUC2-, MUC5AC+, MUC6+, and CDX2-) or I-types (3 cases with MUC1-, MUC2+, MUC5AC \pm , MUC6-, and CDX2 \pm ), respectively The remaining 4 cases either had no positivity or an immunophenotype supportive of a mixed subtype.

Conclusions: IPMN subtypes diagnosed by morphology that correlated best with their respective immunoprofiles were G and I-types. Additionally, 7/11 morphologically ambiguous cases could be classified using IHC. Our results suggest that routine use of IHC would facilitate better standardization in IPMN subtyping.

\section{Reproducibility of the Morphologic Subtypes of Intraductal} Papillary Mucinous Neoplasm of the Pancreas

Heewon Kwak, Xiuli Liu, Daniela Allende, Rish Pai, John Hart, Shu-Yuan Xiao. University of Chicago, Chicago, IL; Cleveland Clinic Foundation, Cleveland, OH. Background: Intraductal Papillary Mucinous Neoplasm (IPMN) is a premalignant lesion with approximately one-third associated with invasive carcinoma. Four histologic subtypes are recognized: gastric $(\mathrm{G})$, intestinal (I), pancreatobiliary (PB), and oncocytic $(\mathrm{O})$, which are thought to have prognostic value as G-type associated tubular adenocarcinoma has a worse prognosis than I-type associated colloid carcinoma. Given the importance of subtyping IPMN, this study analyzes the interobserver variability of morphologic subtypes of IPMN.

Design: Fifty IPMN cases were selected and reviewed independently by 5 GI pathologists for classification by $\mathrm{H} \& \mathrm{E}$ morphology (G, I, PB, O, or mixed subtypes), grading of dysplasia (low, intermediate, or high), and diagnosing invasion. Interobserver agreement was calculated using the Fleiss' kappa statistic $(\kappa)$. A consensus diagnosis was defined as having at least 4 out of 5 pathologists in agreement. An intradepartmental consensus meeting with 2 of the 5 GI pathologists was held to review cases without an initial consensus diagnosis for IPMN subtype.

Results: The overall $\kappa$ for the morphologic subtyping of IPMN before and after an intradepartmental consensus meeting was 0.40 and 0.43 , respectively. A consensus diagnosis was reached in 35 of 50 cases $(70 \%)$.

Fleiss' Kappa Statistics of IPMN Subtyping

\begin{tabular}{||c||c||c||c||c||c||c||}
\hline IPMN subtype & G & I & PB & O & Mixed1 & Overall \\
\hline \hline \multirow{2}{*}{ א2, Standard error (SE) } & 0.38, & 0.56, & 0.41, & 0.08, & 0.16, & 0.40, \\
& 0.04 & 0.04 & 0.04 & 0.04 & 0.04 & 0.03 \\
\hline \hline \multirow{2}{*}{ K3, SE } & 0.40, & 0.64, & 0.44, & 0.11, & 0.14, & 0.43, \\
& 0.04 & 0.04 & 0.04 & 0.04 & 0.04 & 0.03 \\
\hline
\end{tabular}

1Mixed indicates each subtype present comprises $\geq 25 \%$ of thelesion

$2 \kappa$ value of 0 to

$3 \mathrm{New} \kappa$ after the intradepartmental consensus meeting

The $\kappa$ was 0.42 (SE 0.03) for grading dysplasia and 0.72 (SE 0.04) for diagnosing invasion.

Conclusions: The interobserver reproducibility of subtyping IPMN by morphology was demonstrated as having moderate agreement among GI pathologists with a consensus diagnosis in $70 \%$ of the cases. Classifying I-type by morphology had the best reliability among all of the IPMN subtypes. Grading dysplasia and diagnosing invasion had 
moderate agreement and good agreement, respectively. Morphologic evaluation alone is likely insufficient in subtyping about $30 \%$ of IPMNs and ancillary tests may increase interobserver agreement.

\section{The Diagnostic Value of ERCP FNA Biopsy in Pancreatic} Neuroendocrine Tumors (PNETs) and Its Grading

Lisa Laskiewicz, Xiaofei Wang. UMass Medical School, Worcester, MA.

Background: Pancreatic neuroendocrine tumors are uncommon, but increased detection through imaging and study of these tumors has led to better understanding of their biologic behavior, which resulted in new diagnostic terminology and grading system by the WHO in 2010. Most PNETs are diagnosed by fine needle aspiration (FNA) biopsy. Grading of these tumors has important therapeutic implications. Our goal is to examine whether grading on ERCP FNA biopsy cases correlates with grading on surgical resection specimens.

Design: Surgical cases of pancreatic resections for PNETs diagnosed from 1998 to 2014 at our institution were retrieved. Cases with a diagnostic FNA prior to resection were included. Tumors were graded on FNA material and on surgical sections with evaluation of Ki-67 according to the 2010 WHO criteria. Dark-brown nuclear staining was considered positive, with grade I tumors exhibiting staining in $£ 2 \%$ of cells and grade II tumors exhibiting staining in $3-20 \%$ of cells.

Results: Our search yielded 26 cases of PNETs that had a surgical resection with corresponding FNA. The patient population had a median age of diagnosis of 57.1 years and was comprised of $65.4 \%$ females and $34.6 \%$ males. Of 26 cases, 2 had FNAs that were nondiagnostic. The 24 cases evaluated included 14 cases of grade tumors and 10 cases of grade II tumors on surgical resection, with all cases diagnosed as PNET on FNA. Evaluation of grading between tumor resections and FNA specimens revealed that 21/24 (87.5\%) FNA cases had Ki-67 results comparable to the surgical resections, thus allowing for an accurate diagnosis of grade. Direct comparison of grade I versus grade II diagnoses revealed that FNA correctly detecting grade I tumors has a positive predictive value (PPV) of $86.7 \%$, sensitivity of $92.9 \%$ and specificity of $80.0 \%$. Correct FNA diagnosis for grade II tumors has a PPV of $88.9 \%$, sensitivity of $80.0 \%$ and specificity of $92.9 \%$. Of the 3 cases with incompatible Ki-67 staining, $2 / 24(8.3 \%)$ cases had an FNA diagnosis of grade I when the final resection diagnosis revealed grade II tumor; $1 / 24(4.2 \%)$ case had an FNA diagnosis of grade II when the final resection diagnosis revealed grade I tumor.

Conclusions: PNETs are usually diagnosed by FNA, which helps to guide surgical management. Different management may occur between grade I and II PNETs. Grading of PNETs on FNA, including the use of Ki-67, should always be assessed as there is good correlation between the diagnostic grade on FNA and on surgical resection. Given the diagnostic value of FNA for PNETs, it is useful to report the grading on FNA diagnosis to clinicians.

1782 High DBC1 (CCAR2) Expression Is a Favorable Prognostic Marker in Gallbladder Carcinoma

Juhie Lee, Kyu Yeoun Won, Byeong-Joo Noh, Gou Young Kim, Sung-Jig Lim, Hee Seung Choi, Yong-Koo Park, Youn Wha Kim. Kyung Hee University Hospital, Seoul, Korea; Kyung Hee University Hospital at Gangdong, Seoul, Korea.

Background: Deleted in breast cancer 1(DBC1, renamed CCAR2 (cell cycle activator and apoptosis regulator 2)) is a protein which plays a role in the regulation of cellular metabolism, transcription, and stress response. In regards to cancer, it has been debating whether $\mathrm{DBC} 1$ is a tumor suppressor or tumor promoter. $\mathrm{DBC} 1$ is over-expressed and correlates with poor prognosis in some human cancers, but its role in gallbladder cancer remains unknown. This study aims to evaluate expression of $\mathrm{DBC} 1$ and its prognostic values in 104 patients of gallbladder cancer.

Design: Formalin-fixed paraffin-embedded (FFPE) tissue samples from 104 patients with gallbadder cancer were obtained in Korea. Immunohistochemical analysis of DBC1 was performed and measured using proportion and staining intensity.

Results: Immunohistochemial staining showed high expression of DBC1 in $31 \%$ $(32 / 104)$ and low expression in $69 \%(72 / 104)$ of gall bladder carcinoma, compared with the adjacent non-tumor tissues. High DBC1 expression was significantly correlated with favorable clinico-pathologic variables including low histologic grade, low TNM stage and absence of lymph node metastasis and lymphatic invasion. Furthermore, high-DBC1 expression group showed a better overall survival rate compared to the low-DBC1 expression group $(\mathrm{p}=0.02)$

Conclusions: Our findings suggest that $\mathrm{DBC} 1$ has been involved in tumorigenesis of gall bladder cancer and its expression level may be used as an independent good prognostic factor

1783 Needle-Based Confocal Laser Endomicroscopy: Establishment of Diagnostic Criteria, Based on Correlated Histologic Features, Helping in the Diagnosis of Pancreatic Cysts

Anne Isabelle Lemaistre, Bertrand Pujol, Marc Giovannini, Fabrice Caillol, Laurent Palazzo, Bertrand Napoleon. Centre Leon Berard, Lyon, France; Hopital Privé Jean Mermoz, Lyon, France; Institut Paoli Calmettes, Marseille, France; Clinique du Trocadero, Paris, France.

Background: The nature of pancreatic cysts often remains uncertain, despite several tests including FNA cytology. nCLE is a new technique enabling microscopic observation during EUS-FNA procedures. A french study (CONTACT), based on correlations with histologic features, aims at establishing nCLE criteria for the characterization of serous cystadenomas (SCA), mucinous cystadenomas (MCA), Intraductal papillary mucinous neoplasms (IPMNs) and pseudocysts (PC).

Design: Between June 2012 and Septembre 2014, 67 patients were enrolled. Final diagnosis was considered as stringent in case of histological analysis of the surgical specimen and/or FNA results was undoubtedly positive. For the other cases, the diagnosis was obtained from an adjudication committee including two pathologists.

There were 33 SCA (14 proven by undoubtedly histo cytological analysis, 21 by consensus), 8 MCA ( 7 proven by surgery, 1 by consensus), 20 IPMNs ( 3 proven by undoubtedly histo cytological analysis, 17 by consensus) and 6 PC (1 proven by surgery, 5 by consensus)

All the nCLE videos have been reviewed by four gastroenterologists and two pathologists in an unblinded manner. Histological images of 21 cystic lesions extracted from an archive database were reviewed simultaneously in order to identify specific nCLE criteria.

Results: SCA presented with a vascular network in the superficial layer of the cyst wall. It is correlated with its particular histologic vascular organization.

IPMNs presented with a papillae, which is characterized by two epithelial borders surrounding a bright vascular flow.

In MCA, the epithelial border lining the cyst wall was furtively and recurrently seen, without characteristic papillary organization.

Pseudocysts presented with a field of bright, grey and black particules from 10 to 40 microns corresponding to inflammatory cells.
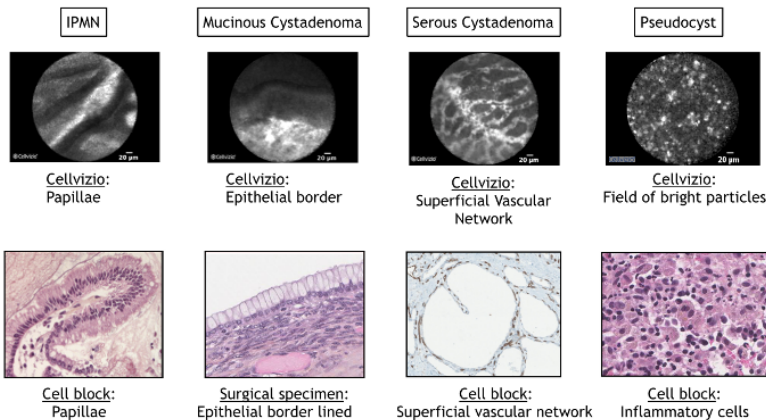
Epithelial border lined
fibrous vascular cyst wall
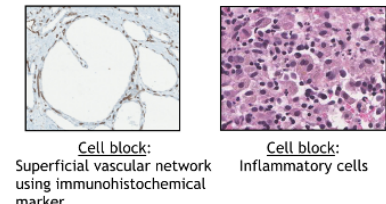

marker

Conclusions: nCLE images obtained in cystic pancreatic tumors can be explained by histological features. These new criteria could help in the differential diagnosis of pancreatic cyst. Pathologists have to be implicated in CLE interpretation to increase the confidence in this new technic.

1784 High-Quality Whole Genome Sequencing (WGS) of Pancreatic Adenocarcinoma Using Systematic Laser Capture Microdissection (LCM) and Flow Sorting (FS)

Sheng-Ben Liang, Faiyaz Notta, Tiera Dookhie, Andrew Brown, Parisa Sairafi, Timothy Beck, Julie Wilson, Dianne Chadwick, Lincoln Stein, John McPherson, Steve Gallinger Michael Roehrl. University Health Network, Toronto, ON, Canada; Ontario Institute for Cancer Research, Toronto, ON, Canada.

Background: Pancreatic ductal adenocarcinoma (PDAC) remains a very aggressive disease with few promising treatment options. WGS provides a powerful platform for deep insight into disease-related and therapy-relevant genomic alterations. Due to often extremely low tumor cellularity, heterogeneity, and desmoplasia, WGS of primary $\mathrm{PDAC}$ is a formidable challenge. We illustrate an integrated enrichment workflow that provides very high-quality WGS data from primary PDAC.

Design: LCM was performed on frozen tumor samples (typically 200,000 tumor cells per NGS library), while FS was used with fresh tissue (EpCAM positive selection). Sequencing was performed on HiSeq2000/2500 instruments at 50× (tumor) and 35× (germline) depth.

Results: Whole genome sequencing of 102 fully tumor-cell enriched PDACs has been completed. In the LCM-enriched cohort, the per patient median number of observed whole genome SNV was 5278 , the median number of non-synonymous exonic mutations was 38 , and the median number of indels was 456 . In the flow-enriched cohort, the median per patient observed whole genome SNV was 5866, the median number of non-synonymous exonic mutations was 41 , and the median number of indels was 537. The most commonly mutated genes were KRAS (90\%), TP53 (55\%), SMAD4 (10\%). Importantly, however, there was a long tail of mutated genes $(\sim 50)$, each of which is mutated in $5-8 \%$ of patients (e.g., MUC16, ARID1A, CDKN2A), highlighting the degree of genomic heterogeneity within PDAC. A small subset $(n=5)$ of PDACs in our cohort show a "hypermutated" phenotype (>300 non-silent exonic mutations or $>20,000$ mutations in the genome). Chromotryptic (large-scale genome rearrangement) events also appear commonly ( $50-60 \%$ of cases) and may be associated with $\mathrm{p} 53$ status. A mutational signature analysis tentatively identified at least 4 distinct genomic PDAC subtypes.

Conclusions: Systematic use of LCM and FS prior to WGS has significantly improved the ability to call somatic mutations, and perform copy number and structural analyses. PDAC is a genomically heterogeneous disease. Pathway mapping of the alterations is under way. LCM and FS coupled with WGS is a powerful combination that would also be applicable to other cancers that are characterized by high intrinsic heterogeneity and/or low cancer cell content but also to the study of cancer-associated non-neoplastic compartments (such as tumor stroma). 
1785 Ampullary Biopsy Morphology Combined With a Three Biomarker Panel Is a Unique Approach To Distinguish Adenomas and Adenocarcinomas From Reactive Epithelial Change: A Large Cohort Study Amy Lo, Haonan Li, Xiaoming You, Yihe Yang, Jie Liao, Nike Beaubier, MS Rao, Guang-Yu Yang. Northwestern Memorial Hospital, Chicago, IL.

Background: Differentiation of reactive epithelial change, adenoma and adenocarcinoma in ampullary biopsy based on morphology alone is challenging. Accurate diagnosis could be determined by combining a panel of biomarkers with morphologic features to more clearly separate subtle morphologic changes. Therefore, we identified 383 ampullary biopsies, reviewed their morphologic features and established whether a panel with three biomarkers including p53, HMGA2 and Ki-67 could help differentiate adenoma and adenocarcinoma from reactive epithelial change. Design: Ampullary biopsies obtained between 2003-2014 were identified from our institutional database $(\mathrm{n}=383)$. All cases were categorized into benign, reactive change, adenoma, adenocarcinoma or other based on morphology alone. Immunohistochemistry (IHC) for p53, Ki-67 and HMGA2 were performed on randomly selected slides from each category with appropriate controls. Epithelial cell nuclear positivity from 5 high power fields was determined for each biomarker and cases with more than 5\% positive epithelial cells were considered positive results.

Results: Normal ampullary mucosa was seen in $24 \%$ of cases $(n=91)$ while $45 \%$ had reactive epithelial change $(\mathrm{n}=171), 20 \%$ were adenomas $(\mathrm{n}=77), 10 \%$ were adenocarcinomas $(n=40)$ and $1 \%$ were other $(n=4)$. Cases with reactive epithelial change demonstrated inflammation and/or fibrosis $(\mathrm{n}=136)$, pseudostratefication and hyperchromasia similar to adenomas $(\mathrm{n}=41)$, or cytological atypia suspicious for adenocarcinoma $(\mathrm{n}=19)$. $\mathrm{p} 53$ was positive in $75 \%$ of adenocarcinoma cases, but negative in cases with adenoma or reactive epithelial change. Ki-67 was diffusely positive in the proliferative zone with extension to the luminal surface in all adenoma cases (topdown growth pattern), but was limited to the epithelial proliferative zone in all cases of reactive epithelial change. HMGA2 was positive in $80 \%$ of adenocarcinomas, $8 \%$ of cases with reactive epithelial change and none of the adenomas.

Conclusions: We demonstrate that morphology together with a three biomarker panel is a unique approach to differentiate reactive epithelial change from adenoma and adenocarcinoma in limited ampullary biopsy material. Malignant biomarkers p53 and HMGA2 are useful and sensitive in diagnosis of ampullary adenocarcinoma while Ki-67 highlights a top-down growth pattern in adenomas to distinguish them from reactive epithelial change.

1786 Clinicopathologic Analysis of 21 Deceptively Benign-Appearing Adenocarcinomas ("Adenoma Malignum") of the Gallbladder and Their Differential Diagnosis From Pseudoinfiltrative Processes

Bahar Memis, Brian Quigley, Michelle Reid, Nevra Dursun, Gizem Akkas, Selda Karaaslan, Olca Basturk, Burcu Saka, Pelin Bagci, Serdar Balci, Alyssa Krasinskas, Juan Roa, Oscar Escalona, Juan Sarmiento, Volkan Adsay. EU, Atlanta, GA; Wayne State University, Detroit, MI; PUC, Santiago, Chile.

Background: Exceptionally well-differentiated (EWD) gallbladder carcinomas (GBC) are highly challenging diagnostically.

Design: Among 656 GBCs, 21 with EWD pattern constituting $>75 \%$ of the tumor were analyzed.

Results: Clinical: Mean age $=64$ (same as ordinary GBC). $\mathrm{F} / \mathrm{M}=5.6$ (vs 3.9). $76 \%$, had pT2 (vs 34\%). Pathology: Well formed tubules with open round lumina, some with irregular contours; often similar to mammary tubular ca; axis often parallel/oblique to the surface; lined by polarized cytologically-bland cells, some with apocrine snouts, occurred in 5 patterns, often mixed: 1) Flattened cells (seen in 17 cases): minimal cytoplasm, flattened nuclei, often forming cystic/syringoid pattern (Fig. A). 2) Round nuclei (in 15): basal/suprabasal, large, round, uniform cuboidal nuclei with prominent nucleoli; moderate cytoplasm, some clear, some acidophilic (Fig. B). 3) Tall columnar (in 8): gastric/endocervical-like; nuclei compressed at the periphery (Fig. C). 4) Grooved-nuclei (in 6): minimal/moderate cytoplasm; visible nuclei with uniform chromatin but subtle grooves (Fig. D). 5) PTC-like crowded nuclei (in 3): overlapping, elongated, hypo-chromatic nuclei with high N/C. Differential Diagnosis: 1) AschoffRokitansky sinuses, which, unlike EWDs, formed compressed units perpendicular (and often connecting) to the surface, undulating contours, columnar cells with acidophilic cytoplasm and basal/suprabasal usually elongated nuclei with dense, homogenous chromatin, without nucleoli. 2) Proliferative metaplastic glands. Small, round, clustered (not dispersed), relatively uniform glands with small lumina; some with pyloric mucin uniform nuclei; fine chromatin. F/U: Median surv. was 26 mos. 11 (55\%) rapidly died of disease (at a median of 6 mos). Of the $9(45 \%)$ that were alive, 5 lived $>10$ yrs, 3 of whom had very limited $\mathrm{T} 2 \mathrm{ca}$.

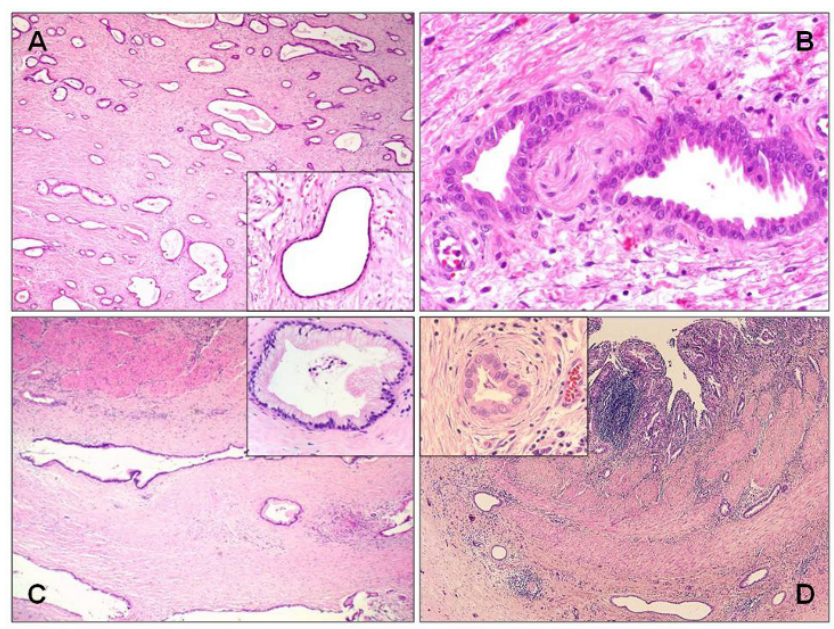

Conclusions: Recognition of the patterns elucidated in this study can be crucial in the challenging differential diagnosis of extremely well differentiated GBC from their benign mimics. Although low grade, these are still aggressive with a median survival of $\sim 2$ years. However, patients with low stage may experience long-term survival.

1787 Claudin Expression in Well Differentiated Pancreatic Neuroendocrine Tumors

Rashna Meunier, Shamlal Mangray, Chaohui Zhao, Kara Lombardo, Lelia Noble, Shaolei Lu, Murray Resnick, Evgeny Yakirevich. Rhode Island Hospital, Providence, RI. Background: Well differentiated neuroendocrine tumors (WDNETs) of the pancreas, gastrointestinal system, and lung are histologically similar. Islet 1 and PAX8 are considered markers of pancreatic WDNETs; however, they are also expressed in rectal WDNETs. Claudins are a major component of the tight junctions that are present in both normal and neoplastic epithelial tissues. We investigated whether claudins $1,3,4,7$, and 8 may help in distinguishing pancreatic from rectal and small intestinal (SI) WDNETs. Design: Eighty-six cases of WDNETs (grade 1-2) that included 17 pancreatic, 12 rectal, and 57 SI were studied. Tissue microarrays were analyzed for immunohistochemical expression of claudins $1,3,4,7,8$, Islet 1 , and PAX8. Immunoreactivity was assessed based on a combined score of the extent and intensity of staining on a scale of $0-3+$

Results: Normal adult pancreatic islet cells were positive for claudins 3 and 7, but negative for claudins 1,4 , and 8 . Normal pancreatic acinar cells and ducts expressed membranous staining with all claudins studied. In WDNETs the claudin staining pattern was membranous, independent of location. All pancreatic WDNETs were strongly positive for claudin 7 , and displayed immunoreactivity with claudins $3(76.5 \%), 1$ $(47 \%), 8(47 \%)$, and less frequently claudin $4(29 \%)$. Frequent immunoreactivity for PAX8 (65\%) and Islet $1(59 \%)$ was also seen in pancreatic WDNETs. The vast majority of SI WDNETs expressed all claudins (89-98\%), but were negative for Islet 1 and PAX8. Rectal carcinoids were positive for claudins 3, 4, and 7 in all cases, and frequently expressed claudins 1,8 , Islet 1 and PAX8. When comparing cases with only strong $(3+)$ claudin 4 staining, all pancreatic WDNETs were negative, while the vast majority of rectal carcinoids $(91.7 \%)$ were positive.

\begin{tabular}{|c|c|c|c|c|c|c|c|}
\hline & \multicolumn{7}{|c|}{ Number (\%) Positive } \\
\hline $\begin{array}{l}\text { Anatomic } \\
\text { site }\end{array}$ & $\begin{array}{c}\text { Claudin } \\
1\end{array}$ & $\begin{array}{c}\text { Claudin } \\
3\end{array}$ & Claudin 4 & $\begin{array}{c}\text { Claudin } \\
7\end{array}$ & Claudin 8 & Islet 1 & PAX8 \\
\hline $\begin{array}{l}\text { Pancreas } \\
(\mathrm{n}=17)\end{array}$ & $8(47)$ & $13(76.5)$ & $5(29)$ & $17(100)$ & $8(47)$ & $\begin{array}{c}10 \\
(59)\end{array}$ & $11(65)$ \\
\hline $\begin{array}{l}\text { Rectum } \\
(\mathrm{n}=12)\end{array}$ & $8(66.7)$ & $12(100)$ & $12(100)$ & $\begin{array}{c}10 \\
(83.3)\end{array}$ & $10(83.3)$ & $\begin{array}{c}10 \\
(83.3)\end{array}$ & $\begin{array}{c}10 \\
(83.3)\end{array}$ \\
\hline $\begin{array}{l}\text { Small } \\
\text { intestine } \\
(\mathrm{n}=57)\end{array}$ & $51(89)$ & $51(89)$ & $55(91)$ & $57(96)$ & $56(98)$ & 0 & 0 \\
\hline
\end{tabular}

Conclusions: This study is the first to comprehensively examine claudin expression in pancreatic WDNETs. Pancreatic WDNETs lack strong claudin 4 expression in contrast to rectal WDNETs. Islet 1 and PAX8 are frequently expressed in rectal carcinoids, limiting their specificity for pancreatic WDNETs. However, the Islet 1+/PAX8+/claudin 4- immunophenotype appears to be specific for pancreatic WDNETs.

1788 Histological Characteristics and the Specificity of Pancreatic Lobular Inflammation in Type 2 Autoimmune Pancreatitis - Comparison With the Inflammation Around Pancreatic Ductal Adenocarcinoma

Kenji Notohara, Lizhi Zhang, Yoichiro Kobashi, Thomas Smyrk. Kurashiki Central Hospital, Kurashiki, Japan; Mayo Clinic, Rochester, MN; Tenri Hospital, Tenri, Japan. Background: Because of a lack of unique clinical features, a histological examination is essential for diagnosing type 2 autoimmune pancreatitis (AIP). The diagnostic hallmark of type 2 AIP is neutrophilic infiltration in the duct epithelium and/or lumens (granulocytic epithelial lesion; GEL). However, this finding is only rarely obtained in biopsy tissues, and thus biopsy diagnosis of type 2 AIP depends partly on lobular inflammatory changes of the pancreas, which have not been discussed in detail to date. 
We compared the histological features of resected type 2 AIP and pancreatic ductal adenocarcinoma (PDA) in order to clarify histological characteristics and the specificity of lobular inflammation in type 2 AIP.

Design: Resected specimens of type 2 AIP (15 cases) and PDA (47 cases) were selected for the study. The histological patterns in type 2 AIP were classified based on types of the predominant epithelial changes [A: remaining of acinar cells, B: acinar-ductal metaplasia (ADM), C: loss of the majority of the epithelium] and types of stroma and inflammatory infiltration ( 0 : edema without inflammatory cells, 1 : edema with inflammatory cells, 2 : fibrosis). For each case, the first and second most prominent patterns were recorded. Types of inflammatory cells and GEL in the intralobular ducts were also observed. Cases with PDA were then examined to find similar histological features.

Results: Among the histological patterns, the B-1 pattern was the most significant (seen in 13 cases), and was usually associated with the C-1 pattern. Neutrophilic infiltration was observed in all cases, notably in the areas with $\mathrm{ADM}$, but the degree of the intensity was varied. There was one case of the $\mathrm{C}-2$ and $\mathrm{C}-1$, and $\mathrm{C}-1$ and $\mathrm{A}-0$ patterns, respectively. GEL in the intralobular ducts was observed as prominent in 3 cases, and mild in 9. In the former cases, the duct epithelium was so severely disrupted that the recognition of GEL was often difficult. The B-1 pattern was also common in PDA cases, being identified in $27(57 \%)$ out of 47 cases. This finding was most often seen at the infiltrative margin of PDA.

Conclusions: ADM with neutrophilic infiltration was the predominant pattern seen in the lobules of type 2 AIP. Although this finding resembles GEL, ductal metaplasia is a pathological structure, and thus distinct from GEL, which affects pre-existing ducts. ADM with neutrophilic infiltration is not specific, and is commonly seen even in cases of PDA.

1789 Diagnostic Impact of Intratumoral Heterogeneity of Ki67 Labeling Index in Pancreatic Neuroendocrine Tumors (NETs): Analysis of 91 Cases Shows Hot Spot Counting Correlates Better With Signs of Aggressiveness Than Counting Random Areas

Michelle Reid, Nobu Ohike, Olca Basturk, Sergei Klimov, Pelin Bagci, Gizem Akkas, Volkan Adsay. Emory University, Atlanta, GA; Showa University, Showa, Japan; Memorial Sloan Kettering Cancer Center, New York, NY; Georgia State University, Atlanta, GA

Background: Although intratumoral heterogeneity in Ki67 labeling index has been noted in pancreatic NETs, it's effect on tumor grade in hot spots versus random areas within the tumor has not been systematically assessed.

Design: Ki67 labeling index (KI) was calculated in 91 pancreatic NETs, 29 of which had metastatic tumors available for assessment. Minimum of 2000 cells were counted both at hot spots $(\mathrm{KI}(\mathrm{h}))$ and in random areas $(\mathrm{KI}(\mathrm{r}))$ using the camera-captured photograph method.

Results: There were 42 M, 49 F, mean age 54 yrs (23-85yrs), 9 multifocal, 24 functional and 6 hereditary (4 MEN, 2 VHL) NETs of mean size $4.1 \mathrm{~cm}$ (range $0.6-17 \mathrm{~cm}$ ). There was striking heterogeneity in the intra-tumoral distribution of Ki67 labeling. Mean KI was $2 \mathrm{X}$ higher in hot spots $(5 \%$, range $0.3-23.7 \%)$ than random areas $(2.1 \%$, range $0.1-18.7 \%$ ) but intriguingly no significant difference was found between primary and mets. The agreement between $\mathrm{KI}(\mathrm{h})$ and $\mathrm{KI}(\mathrm{r})$ in calculating grade of NETs was low (weighted kappa $(K)$ 0.2770). In fact more than twice as many G2NETs were identified by counting hot vs random spots, while random counting missed all G3 NETs.

\begin{tabular}{|c|c|c|c|c|}
\hline ENETs/WHO & \multicolumn{2}{|c|}{ KI(h) vs KI(r) } & \multicolumn{2}{|c|}{$\mathrm{KI}(\mathrm{h})$ vs Mitotic Index } \\
\hline G1( & 43 & 76 & 43 & 82 \\
\hline $\mathrm{G} 2(3-20 \%)$ & 44 & 15 & 44 & 9 \\
\hline G3 $(>20 \%)$ & 4 & 0 & 4 & 0 \\
\hline Kappa (K) & \multicolumn{2}{|c|}{0.277 (C.I. $0.1582-0.3958)$} & \multicolumn{2}{|c|}{0.126 (C.I. $0.0224-0.2302$ ) } \\
\hline
\end{tabular}

As a result $33 / 91$ cases $(36 \%)$ were placed in a different grade based on whether $\mathrm{Ki}(\mathrm{h})$ or $\mathrm{Ki}(\mathrm{r})$ was used for grading $(\mathrm{p}<0.001)$. The agreement between $\mathrm{KI}(\mathrm{h})$ and mitotic index was also weak $(K, 0.126)$. Most importantly $\mathrm{KI}(\mathrm{h})$ was more predictive of metastasis $(\mathrm{p}=0.015)$ and tumor size $(\mathrm{p}=0.0006)$ than $\mathrm{KI}(\mathrm{r})(\mathrm{p}=0.0712$ and $\mathrm{p}=0.07$ respectively). For tumors $>5 \mathrm{~cm} \mathrm{KI}(\mathrm{h})$ trended toward predicting perineural invasion $(\mathrm{PNI})(\mathrm{p}=0.055)$ but KI(r) did not ( $\mathrm{p}=0.089)$.

Conclusions: There is significant intratumoral heterogeneity in Ki67 distribution in primary pancreatic NETs, which, depending on sampling and area counted may lead to underestimation of grade in $36 \%$ of cases. This study supports the current approach of using hot spots as the grade parameter, by illustrating a stronger association of hot spot count with signs of aggressiveness such as metastatic risk, tumor size and PNI, than random. This has significant implications for KI calculation in biopsies and cytology samples.

1790 Mismatch Repair Status as a Predictive Marker in Pancreatic Ductal Adenocarcinoma

Maziar Riazy, Steve Kalloger, Brandon Sheffield, Hector Li-Chang, Charles Scudamore, Daniel Renouf, David Schaeffer. University of British Columbia, Vancouver, BC, Canada; Pancreas Centre, Vancouver, BC, Canada.

Background: Deficiencies in DNA mismatch repair (MMR) have been associated with improved survival but inferior response to some forms of chemotherapy, especially in the case of fluoropyrimidine analogs and colorectal cancer. However, there are few and conflicting reports addressing the prognostic and predictive value of MMR status in pancreatic ductal adenocarcinoma (PDAC), which is predominantly treated with pyrimidine analogs.

Design: A tissue microarray with associated clinical outcome data, comprising 236 surgically-treated PDAC patients from Vancouver General Hospital (1998 - 2014), was stained for four MMR enzymes (MLH1, MSH2, MSH6 and PMS2). MMR-deficiency (MMR-d) was defined as an absence of nuclear staining in tumor cells for any of the MMR enzymes, while presence of nuclear staining was interpreted as a MMR-proficient (MMR-p) profile. Univariable disease-specific survival (DSS) was assessed using the Kaplan-Meier method, while Cox-Proportional Hazards Model was performed to calculate multivariable DSS.

Results: Of the 236 patients, 77 (33\%) were treated with an adjuvant pyrimidine analog and $58(25 \%)$ showed a MMR-d immunoprofile, while $178(75 \%)$ were MMR-p. In the MMR-p cohort, adjuvant chemotherapy, pathological stage, perineural invasion and the presence of tumor budding were significant prognostic variables of DSS; however, no difference existed amongst the two cohorts with regard to these variables. The distribution of MMR profiles in relation to adjuvant chemotherapy and median DSS are shown in Figure 1
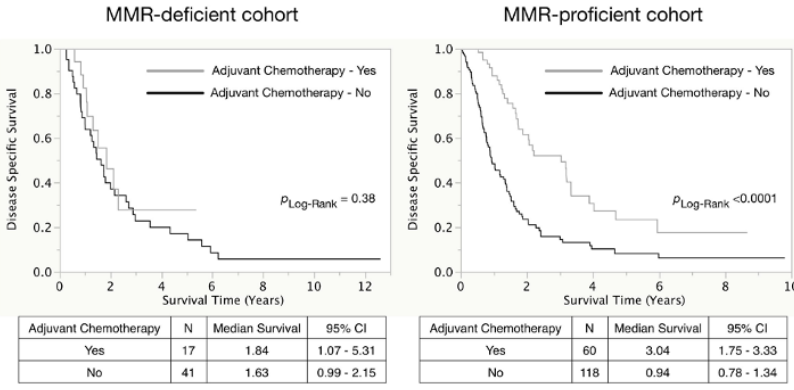

Conclusions: In our cohort, adjuvant chemotherapy with a pyrimidine analog conferred no advantage to MMR-d PDAC patients. MMR immunoprofiling appears to lend itself as a feasible step to guide therapy in PDAC and prospective evaluation of this finding is warranted.

1791 Elafin Expression in Gastrointestinal Neoplasia and Correlation With Sensitivity To PI3K/mTOR Inhibitor

Avi Rosenberg, Holger Pflicke, Jennifer Dreiling, Zeng-Feng Wang, Kris Ylaya, Stephen Hewitt, Udo Rudloff, Markku Miettinen. Johns Hopkins University, Baltimore, MD; National Cancer Institute, Bethesda, MD.

Background: Overexpression of ELAFIN (serine protease inhibitor of elastases and neutrophil proteinase 3 ) in a variety of malignancies including carcinomas and glioblastoma has been reported. Proposed mechanisms that induce ELAFIN overexpression include hypoxia and its location on a commonly occurring amplicon (20q12-13.1). Published reports suggest that elafin expression impacts outcome in a tumor specific manner, i.e. correlation with good prognosis in breast carcinoma vs poor prognosis in glioblastoma and decreased sensitivity to genotoxic agents in high-grade serous ovarian carcinomas. We report ELAFIN expression across a broad spectrum of carcinomas and identify malignancies with previously unreported expression.

Design: ELAFIN expression by immunohistochemistry was scored in 1,302 tumors (26-147 tissues/tumor subtype). All tissues were collected under IRB approved protocols and anonymized.

Results: We observe strong positivity for ELAFIN in a subpopulation of carcinomas of the bladder $(18 \%)$, breast $(5 \%)$, cholangiocarcinoma $(32 \%)$, colorectal $(54 \%)$, endometrial (37\%), gastric (35\%), hepatocellular $(2.6 \%)$, lung adenocarcinoma $(7.5 \%)$, mesothelioma (3\%), ovarian $(20.7 \%)$, pancreatic $(56 \%)$ and renal cell $(2 \%)$ as well as the expected positivity in various squamous cell carcinomas (41-76\%). No melanomas, basal cell carcinomas or papillary thyroid carcinomas with strong expression were identified. A significant correlation of elafin mRNA levels and sensitivity to the PI3K/mTOR inhibitor NVP-BEZ235 in a large panel of pancreatic cancer cell lines was observed (Fisher's exact test, $\mathrm{p}=0.0161$ ). Pathway analysis of genes overexpressed in elafin-high cells suggests active roles of PTGS2 (COX-2), NFkB-complex and PI3K signaling. Conclusions: Aberrant ELAFIN expression is observed in a previously unreported spectrum of adenocarcinomas, including a subset of gastrointestinal adenocarcinomas. elafin's genomic location is within an amplicon that contains numerous oncogenes associated with the progression of colorectal adenomas to adenocarcinomas and metastatic potential and is frequently observed in intrahepatic cholangiocarcinomas (67\%), colorectal carcinomas ( $>70 \%$ in HNPCC), gastric carcinomas (up to $77.8 \%$ ), pancreatic (up to $100 \%$ in some subtypes). Preliminary data in pancreatic cancer cells support a possible role for elafin expression as indicator for a response to targeted therapies. Further studies are necessary to corroborate and elucidate whether this correlates with progression and is informative in predicting response to genotoxic agents in the other malignancies.

\section{NGS Sequencing Augments Cytologic Evaluation of Pancreatic} Cyst Fluids

Ali Sakhdari, Parnian Ahmadi Moghaddam, Chi Young Ok, Keith Tomaszewicz, Ediz Cosar, Lloyd Hutchinson. Umass Memorial Medical center, Worcester, MA.

Background: Advances in diagnostic imaging have led to a large increase in the detection of pancreatic cystic lesions. An accurate diagnosis is needed to manage the patient and relies on cytologic examination and tumor marker analysis of cyst fluid which may have limitations. The aim of this study is to evaluate the diagnostic utility of gene mutation analysis in cytologic samples of pancreatic cystic lesions.

Design: From 2008 to 2014, we prospectively evaluated 211 patients (101 male, 110 female, median age 61) with pancreatic cyst(s) who underwent endoscopic ultrasound (EUS) guided fine needle aspiration (FNA) of cyst fluid. Initially, KRAS and GNAS 
status was tested by quantitative peptide nucleic acid (PNA) clamp PCR in 207 and 54 cases respectively. Mutations were confirmed by Sanger sequencing. A subset of 20 cyst fluids were analyzed by ion torrent sequencing for KRAS, GNAS, BRAF, PIK3CA, VHL, CTNNB1, TP53 and SMAD4 mutations. Both methods can detect a $1 \%$ mutation frequency. DNA quality/quantity was evaluated using multiple methods. Cyst fluid CEA and amylase levels were also measured. Clinical follow-up data was obtained. Results: Cytology diagnoses were classified into four groups: non-diagnostic (ND, group 1,24\%), negative (NEG group 2, 56\%), atypical/suspicious (group 3,17\%), and positive for malignancy (group 4, 4\%). Three samples (1.4\%) failed mutation analysis. Mutation frequency was as follows: KRAS $(38 \%, 81 / 211)$, BRAF V600E $(0.5 \%, 1 / 211)$, GNAS $(20 \%, 11 / 54)$, VHL $(10 \%, 2 / 20)$. In cases with GNAS activating mutation, $73 \%$ $(8 / 11)$ were also KRAS mutated. Mutational analysis of cytology groups showed the following. Group 1: KRAS $(40 \%, 20 / 50)$, BRAF $(0 \%, 0 / 45)$, GNAS $(38 \%, 3 / 8)$, VHL $(33 \%, 2 / 6)$. Group2: KRAS $(20 \%, 23 / 116)$, BRAF $(1 \%, 1 / 111)$, GNAS $(12 \%, 4 / 33)$, VHL $(0 \%, 0 / 11)$. Group3: KRAS $(85 \%, 29 / 34)$, BRAF $(0 \%, 0 / 29)$, GNAS $(50 \%, 4 / 8)$, VHL $(0 \%, 0 / 3)$. Group4: KRAS $(83 \%, 5 / 6)$, BRAF $(0 \%, 0 / 6)$, GNAS $(0 \%, 0 / 3)$, VHL $(0 \%, 0 / 0)$. Surgical resection was performed in 27 patients. Among 18 KRAS mutated cases, there were 8 intraductal papillary mucinous neoplasm (IPMN), 6 mucinous cystic neoplasm (MCN), 1 PaNIN-3, 2 adenocarcinoma and 1 chronic pancreatitis (CP). Among 9 cases with wild-type KRAS, there were 2 IPMN, 4 MCN, 1 SCA, and 2 CP. Of 6 surgicals with prior GNAS analysis, 3 were KRAS/GNAS positive (IPMN) and 3 were KRAS/GNAS negative (MCN, SCA, Pseudocyst)

Conclusions: Mutation analysis is a good adjunct to cytological evaluation of pancreatic cyst fluid, especially for ND/NEG specimens. In our study, KRAS was positive in $50 \%$ of $14 \mathrm{MCN} / \mathrm{IPMN}$ cases with a ND/NEG diagnosis.

\section{Stromal Palladin Expression Is an Independent Prognostic Factor} of Pancreatic Ductal Adenocarcinoma (PDAC)

Daisuke Sato, Tomoko Mitsuhashi, Takahiro Tsuchikawa, Yutaka Hatanaka, Toru Nakamura, Satoshi Hirano, Yoshihiro Matsuno. Hokkaido University Hospital, Sapporo, Japan; Hokkaido University Graduate School of Medicine, Sapporo, Japan.

Background: Recently, it has been clear that tumor microenvironments including cancer associated fibroblasts (CAFs) in the stroma play an important role in PDAC progression. Although remarkable progress in chemoradiotherapy (CRT) for PDAC has been noted, how CAFs relate to the patients' prognosis and the effect of CRT on CAFs have not been fully investigated. The aim of this study was to evaluate the prognostic implication of palladin, one of the actin-associated proteins reported to be a specific marker of CAFs in PDAC, and also to investigate the changes of its expression in PDAC after CRT.

Design: Firstly, to investigate CAFs' prognostic implications, tissue micro arrays (TMA) representing 167 resected PDACs without preoperative treatment were used for immunohistochemical studies (IHC) of palladin, $\alpha$-smooth muscle actin (SMA), and podoplanin. Correlations between the expression levels of these markers and clinicopathological findings were statistically analyzed.

Secondly, to investigate changes in CAFs after CRT, surgically resected specimens of PDACs with $(\mathrm{CF}, \mathrm{n}=19)$ and without $(\mathrm{SF}, \mathrm{n}=21)$ preoperative CRT were studied by IHC and analyzed in the same way.

Results: In TMAs, disease specific survival rate (DSS) at 5 years of all 167 cases was $23 \%$. Seventy-one cases $(42 \%)$ were positive for palladin and had significant lower DSS ( $p=0.043$, Fig. 1). $\alpha$-SMA and podoplanin were positive in 167 cases $(100 \%)$ and in 133 cases $(80 \%)$, respectively (not related with DSS). On multivariate analysis, palladin expression was an independent prognostic factor $(\mathrm{p}=0.019$, risk ratio 1.65 ). $\mathrm{CF}$ group had significant higher DSS than SF group $(\mathrm{p}=0.036)$. Regarding CRT effects in CF, 4 cases corresponded to Evans' grade I, 8 to IIa, 4 to IIb, 0 to III and 3 to IV. Five of 19 in $\mathrm{CF}$ and 16 of 21 in SF were palladin positive. Palladin expression was significantly lower in $\mathrm{CF}$ ( $\mathrm{p}=0.0037)$, and much lower around degenerated tumor cells $(\mathrm{p}=0.017)$. DSS was significantly lower in palladin positive cases in CF subgroup analysis ( $\mathrm{p}=0.014$, Fig. 2 )

Conclusions: Stromal palladin expression is an independent prognostic factor of PDAC. It might also be a prognostic biomarker after CRT in PDAC.
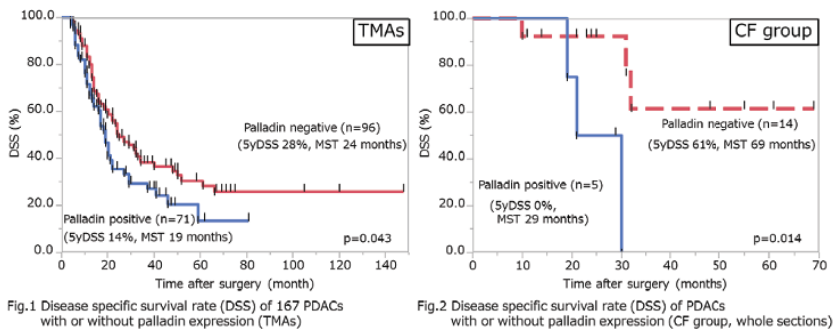

1794 Subclassification of Intraductal Papillary Mucinous Neoplasms: An Institutional Experience and Can We Do it?

Kurt Schaberg, Michael DiMaio, Teri Longacre. Stanford University, Stanford, CA. Background: Intraductal papillary mucinous neoplasms (IPMNs) are subclassified into gastric, intestinal, pancreatobiliary, and oncocytic subtypes based on histologic features. The WHO suggests use of immunohistochemical stains to help subtype IPMNs with ambiguous histology.

Design: H\&E slides from all available IPMN resections at a major academic medical center in the past 5 years were reviewed. Histologic subtyping and grading of dysplasia was performed by 2 GI pathologists. Immunohistochemistry for CDX2, MUC2, and MUC5AC was performed on cases where the histologic subtype could not be determined on routine $H \& E$ sections. These findings were compared to those reported in the primary literature.

Results: 72 IPMNs were identified and subclassified with consensus (Table 1). Eighteen $(25 \%)$ of IPMNs were either unclassifiable due overlapping morphology or contained distinct epithelium from more than one subtype (i.e. "mixed"). Two IPMNs initially unclassifiable strictly by $\mathrm{H} \& \mathrm{E}$ morphology were definitively classified as intestinal after positive staining with CDX2, MUC2, and MUC5AC. Immunohistochemistry for another 7 IPMNs unclassifiable by H\&E did not indicate a clear subtype and often contained discordant results. Immunohistochemistry was not applied to IPMNs with distinct mixed epithelial subtypes on H\&E.

\begin{tabular}{|l||l||l|l||l|l||}
\hline Subtype & Gastric & Intestinal & Pancreatobiliary & Oncocytic & $\begin{array}{l}\text { Unclassfiable/ } \\
\text { Mixed }\end{array}$ \\
\hline \hline No. & $41(57 \%)$ & $8(11 \%)$ & $4(6 \%)$ & $1(1 \%)$ & $18(25 \%)$ \\
\hline Lit. mean & $46 \%$ & $33 \%$ & $17 \%$ & $3 \%$ & \\
\hline Lit. range & $12-72 \%$ & $17-54 \%$ & $1-40 \%$ & $1-13 \%$ & \\
\hline $\begin{array}{l}\text { Associated } \\
\text { withinvasive } \\
\text { carcinoma }\end{array}$ & $7(17 \%)$ & $2(25 \%)$ & $3(75 \%)$ & $0(0 \%)$ & $8(44 \%)$ \\
\hline
\end{tabular}

Conclusions: In our experience, a considerable number of IPMNs were eithe unclassifiable or contained epithelium from more than one subtype. Among those IPMNs initially unclassifiable by H\&E morphology, application of immunohistochemical stains to aid in subtyping allowed for definite classification in only a small subset of cases. Subtyping was particularly difficult in lesions with high-grade dysplasia and almost half of these unclassifiable IPMNs show invasion. As such, definitive classification of such lesions may be unnecessary as the invasive component will determine prognosis and therapy. These data, when taken in context with the significant ranges in the reported prevalence of specific histologic subtypes, suggest that accurate IPMN subtyping is poorly reproducible in up to $25 \%$ of cases and in these problematic cases, immunohistochemistry adds little value.

1795 Intraductal Tubulopapillary Neoplasms of the Bile Ducts: Further Immunohistochemical and Molecular Characterization

Anna Schlitter, Kee-Taek Jang, Burcu Saka, Hyejeong Choi, G Offerhaus, R Hruban, Yoh Zen, Seung-Mo Hong, Bjorn Konukiewitz, Michael Allgaeuer, G Kloppel, M Reid, Irene Esposito, Volkan Adsay, Olca Basturk. TUM, Munchen, Germany; SMC, Seoul, Korea; EU, Atlanta, GA; University Medical Center, Utrecht, Netherlands; Johns Hopkins University, Baltimore, MD; KCH, London, United Kingdom; Asan Medical Center, Seoul, Korea; National Cancer Institute at National Institutes of Health, Bethesda, MD; Wayne State University, Detroit, MI.

Background: Intraductal tubulopapillary neoplasms (ITPNs) are becoming recognized as a distinct tumor type in the pancreatobiliary system.

Design: Twenty ITPNs occurring in the bile ducts were characterized histologically, immunohistochemically and at the molecular level.

Results: General characteristics: $70 \%$ were intrahepatic, and $30 \%$ extrahepatic Mean tumor size was $6.9 \mathrm{~cm}$. All neoplasms had the defining histomorphologic characteristics - they were composed almost exclusively of non-mucinous tubular units with/without sheet-like growth, with no or minimal abortive papilla formation, in addition to comedocarcinoma-like pattern (35\%), clear cell areas $(15 \%)$, oncocytoid cytology (15\%), calcifications/psammomas $(15 \%)$, colloid-like secretions $(10 \%)$, and "mallory-like" hyaline globules (5\%). Invasion was seen in $80 \%$, mostly in the form of tubular adenocarcinoma. With a median follow up of 44 months, 5 -year survival was predicted as $90 \%$. Mucin expression: By definition, none of the neoplasms expressed MUC5AC. MUC1 labeling was seen in $80 \%$ and MUC6 in 30\%, while MUC2 was absent. Immunohistochemical and molecular analysis of common oncogenic pathways: TP53 alterations were rare, detected in $17 \%$ of intraductal components (vs $9 \%$ of invasive). Mutated $K R A S$ as well as mutated $P I K 3 C A$ were identified only in $6 \%$ of intraductal components (vs $0 \%$ of invasive). Similarly, SMAD4/DPC4 inactivation was identified in $7 \%$ of intraductal components (vs $0 \%$ of invasive). No alterations/mutations were identified in $I D H 1, I D H 2, B R A F$, EGFR, HER2 and $\beta$-catenin genes/proteins. Conclusions: Biliary ITPNs have striking similarities to their pancreatic counterpart, both morphologically and immunophenotypically. Despite the relatively high incidence of invasive carcinoma ( $80 \%$ ), limited follow-up suggests that biliary ITPNs, akin to the pancreatic ones, seem to have an indolent behaviour, significantly better than that of conventional cholangiocarcinomas. Also, molecular analyses highlight that alterations of KRAS-TP53 oncogenic signaling pathways, that are commonly mutated in intraductal papillary neoplasm of the bile ducts and conventional cholangiocarcinomas are extremely rare in ITPNs.

\section{Clinicopathologic Characteristics of 136 Mucinous Cystic} Neoplasms

Aristana Scourtas, Jonathan Dudley, William Brugge, Abdurrahman Kadayifci, Mari Mino-Kenudson, Martha Pitman. Massachusetts General Hospital, Boston, MA.

Background: Mucinous cystic neoplasms (MCNs) present a management conundrum. Most are benign but all are resected due to malignant potential. Recent studies have recommended nonsurgical management. We analyzed the preoperative imaging, cytology, and cyst fluid characteristics of 136 histologically confirmed MCNs to assess predictors of a high-risk (HR) cyst for surgical triage.

Design: MCNs resected at the Massachusetts General Hospital between 1991 to present formed the study cohort. Patient demographics, cyst size and mural nodules (MN) by endoscopic ultrasound (EUS), cytology, and cyst fluid CEA levels were correlated with 
histological grade. A low-risk (LR) cyst was defined as low-grade dysplasia (LGD) or intermediate-grade dysplasia (IGD) and a HR cyst as high-grade dysplasia (HGD) or invasive carcinoma (INV) on histology. Performance characteristics were assessed for each parameter, with a cyst size $\geq 3 \mathrm{~cm}$ or MN on imaging and "atypical" or "malignant" cytology considered "true positive" results for predicting malignancy.

Results: Only 15/136 (11\%) of cysts had HR histology. On average, patients with HR cysts were older than those with LR cysts (55 vs. 49, respectively). Imaging had a sensitivity of $50 \%$ and specificity of $57 \%$. Cytology had significantly better performance, with a sensitivity of $67 \%$ and specificity of $80 \%$. The average CEA level $(\mathrm{ng} / \mathrm{mL})$ increased with the grade of dysplasia, but the ranges overlapped between LR and HR cysts (Table 1).

\begin{tabular}{|c|c|c|c|c|}
\hline FEATURE & \multicolumn{2}{|c|}{ LOW-RISK } & \multicolumn{2}{|c|}{ HIGH-RISK } \\
\hline Grade of dysplasia (n) & LGD(96) & $\operatorname{IGD}(25)$ & $\operatorname{HGD}(2)$ & $\operatorname{INV}(13)$ \\
\hline Avg. patient age (range) & $49(16-87)$ & $49(22-76)$ & $64(55-72)$ & $54(36-82)$ \\
\hline $\begin{array}{l}\text { Cyst size, avg. in } \mathrm{cm} \\
\text { (range) }\end{array}$ & $3.2(0.25-9.5)$ & $4.3(1.5-11)$ & $\begin{array}{c}4.1(3.5- \\
4.6)\end{array}$ & $4.5(2.0-6.5)$ \\
\hline Mural nodule(s) n (\%) & $5(9.8)$ & $2(22.2)$ & 0 & $1(25.0)$ \\
\hline Cytology n (\%) & 46 & 9 & 1 & 5 \\
\hline ND/Negative & $37(80)$ & $7(78)$ & $1(100)$ & $1(20)$ \\
\hline Atypical & $9(20)$ & $2(22)$ & 0 & 1(20) \\
\hline Malignant & 0 & 0 & 0 & $3(60)$ \\
\hline $\begin{array}{l}\text { CEA, avg. in } \mathrm{ng} / \mathrm{mL} \\
\text { (range) }\end{array}$ & $\begin{array}{l}2200(0.3- \\
20915)\end{array}$ & $\begin{array}{c}18100(29- \\
87780)\end{array}$ & 500 & $\begin{array}{c}144000(168140- \\
258460)\end{array}$ \\
\hline
\end{tabular}

Table 1. Correlation of pre-operativeparameters with histology

Conclusions: Most MCNs are LR. Cytology is superior to imaging for the prediction of HR MCNs. A MN and/or cyst size $\geq 3 \mathrm{~cm}$ does not correlate with risk. Malignant cytology is specific but insensitive. CEA and amylase do not stratify risk. Other preoperative parameters need to be found to improve accurate preoperative determination of HR MCNs.

1797 ATRX Loss in Pancreatic Neuroendocrine Tumors Is Associated With Disease Recurrence and Decreased Survival

Aatur Singhi, Ta-Chiang Lu, Changqing Ma, Kristi Cressman, Nathan Bahary, Herbert Zeh, Melissa Hogg, Amer Zureikat. University of Pittsburgh Medical Center, Pittsburgh, PA; Washington University, St. Louis, MO

Background: Sporadic, well-differentiated pancreatic neuroendocrine tumor (PanNETs) comprise a heterogeneous group of neoplasms with variable clinical behavior. While multiple prognostic classification systems exist, they do not consider the underlying biology of these tumors. Whole exome studies have identified recurrent mutations in the $\alpha$-thalassemia/mental retardation syndrome X-linked (ATRX) gene, which results in loss of ATRX protein expression, within a subset of PanNETs. ATRX loss was initially reported to be associated with a favorable prognosis; however, more recent studies suggest the opposite. In order to assess the prognostic significance of ATRX loss, immunolabeling for ATRX was performed on a large cohort of PanNETs and correlated with various clinicopathologic factors.

Design: 110 surgically resected PanNETs were immunolabeled for ATRX. Cases were scored as ATRX-negative in the absence of nuclear staining within neoplastic cells, while retained within surrounding non-neoplastic pancreatic parenchyma. Immunostaining results were correlated with patient demographics, pathologic features, disease-free survival (DFS) and disease-specific survival (DSS). The follow-up period ranged from 1.6 to $18.8 \mathrm{yrs}$.

Results: ATRX loss was identified in 11 of $110(10 \%)$ PanNETs with 2 cases demonstrating intratumoral heterogeneity. No significant differences between ATRX status and patient sex $(p=0.19)$, mean tumor size $(p=0.36)$, pancreatic location $(p=$ $0.29)$, histologic grade $(p=0.39)$, perineural invasion $(p=0.21)$, T-stage $(p=0.40)$, $\mathrm{N}$-stage $(p=0.17)$, M-stage $(p=0.11)$, and overall AJCC pathologic stage $(p=0.27)$ was observed. However, ATRX-negative PanNETs did correlate with increased mean patient age (67.2 vs 57.4 yrs, $p=0.02)$, lymphovascular invasion $(p<0.01)$, disease recurrence ( $45 \%$ vs $12 \%, p<0.01)$, shorter mean DFS (5.6 vs 16 yrs, $p=0.001)$ and shorter mean DSS (7.9 vs 17.6 yrs, $p=0.025)$
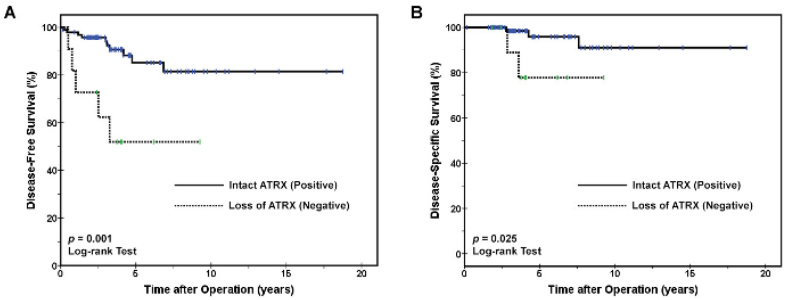

Conclusions: Loss of ATRX protein expression is a poor prognostic factor an associated with disease recurrence and decreased survival in patients with PanNETs after resection. Considering the presence of intratumoral heterogeneity, ATRX loss may be late event within the pathogenesis of PanNETs.
1798 Targeted Six-Gene Panel in the Preoperative Diagnosis and Prognostication of Pancreatic Cysts

Aatur Singhi, Randall Brand, Asif Khalid, Jennifer Chennat, Kenneth Fasanella, Kevin McGrath, Georgios Papachristou, Adam Slivka, N Paul Ohori, Liron Pantanowitz, Herbert Zeh, Amer Zureikat, Melissa Hogg, Kenneth Lee, Allan Tsung, Marina Nikiforova. University of Pittsburgh Medical Center, Pittsburgh, PA.

Background: With advancements in imaging, detection of pancreatic cysts is becoming increasingly common. Analysis of pancreatic cyst fluid by EUS-FNA and ancillary studies, such as CEA, has been particularly useful in cyst classification. But, the inability to predict which cysts can be safely monitored, and which are likely to progress to pancreatic cancer remains challenging. Previous studies have shown molecula analysis of pancreatic cyst fluid to be a promising tool in the diagnosis and prognostic stratification of pancreatic cysts; however, this has not been examined prospectively in the clinical setting.

Design: During a one-year period, 129 pancreatic cyst fluid specimens obtained by EUS-FNA were prospectively submitted for molecular analysis using a 6-gene panel. Hot spot mutations in KRAS, GNAS, TP53, PIK3CA, and PTEN were assessed by next generation sequencing (Ion Torrent PGM). In addition, exons 1 to 3 of $V H L$ were examined by Sanger sequencing. Corresponding cytopathology slides and surgical resection material were reviewed.

Results: Among 129 pancreatic cysts, 30 (24\%) had diagnostic cytopathology and/or follow-up surgical material. These included 5 intraductal papillary mucinous neoplasms (IPMN) with adenocarcinoma, 1 IPMN with high-grade dysplasia (HGD), 3 IPMNs with intermediate-grade dysplasia, 7 IPMNs with low-grade dysplasia (LGD), 1 mucinous cystic neoplasm (MCN) with LGD, 2 serous cystadenomas (SCA), 2 cystic pancreatic neuroendocrine tumors, 2 lymphoepithelial cysts, 1 acinar cell cystadenoma, 5 pseudocysts, and 1 retention cyst. All IPMNs harbored a KRAS and/or GNAS mutation, while TP53 $(\mathrm{n}=4)$ and PIK3CA $(\mathrm{n}=1)$ mutations were only identified in IPMNs with adenocarcinoma. A homozygous rather than a heterozygous mutation in GNAS was seen in the IPMN with HGD. A deletion in PTEN was found in the MCN, and both SCAs had $V H L$ mutations. No mutations were identified in the remaining cysts. Among IPMNs with adenocarcinoma, preoperative cytopathology was suspicious for adenocarcinoma $(\mathrm{n}=2)$, atypical $(\mathrm{n}=2)$ or negative $(\mathrm{n}=1)$. The only adenocarcinoma diagnosed by cytopathology was a retention cyst on resection, which lacked mutations.

Conclusions: Mutational analysis of EUS-FNA obtained pancreatic cyst fluid for $K R A S$ and GNAS, and $V H L$ were highly specific and sensitive for IPMNs and SCAs, respectively. In addition, TP53 and PIK3CA analysis provides a useful adjunct in screening for adenocarcinoma arising in an IPMN. While further studies are required, the 6-gene panel described herein may serve as an important diagnostic and prognostic tool in the assessment of pancreatic cysts.

1799 HSP90 Expression and Antitumoral Activity of Geldanamicin and Its Analog in Gallbladder Carcinoma

Jose Valbuena, Helga Weber, Pamela Leal, Patricia Garcia, Carolina Bizama, Jaime Espinoza, Juan Roa. Facultad de Medicina, Universidad de La Frontera, Temuco, Chile; Pontificia Universidad Católica de Chile, Facultad de Medicina, Santiago, Region Metropolitana, Chile; Johns Hopkins University, Baltimore, MD; Pontificia Universidad Católica de Chile. Facultad de Medicina, Santiago, Region Metropolitana, Chile; Pontificia Universidad Catolica de Chile. Facultad de Medicina, Santiago, Region Metropolitana, Chile.

Background: Gallbladder carcinoma (GBC) is a lethal neoplasia with few therapeutic alternatives. It is the second cause of death by cancer among Chilean women. Heat shock protein (HSP)-90 is a molecular chaperone involve in the stabilization and conformational changes of several oncoproteins. Geldanamicin (GA) and its analog 17-allylamino-17-des-methoxygeldanamycin (17-AAG) have shown to inhibit the action of HSP90 in several malignant tumors. The expression of HSP90 and the antitumoral activity of HSP90 inhibitors have not been studied extensively in GBC.

Design: HSP90 expression was assessed in 6 GBC cell lines and 209 tumors from archival tissue samples by Western Blot and immunohistochemical methods, respectively. The in vitro effects of GA and 17-AAG on cellular viability and migration were evaluated by MTS and Transwell migration assays. The potential therapeutic effect of 17-AAG was evaluated by a subcutaneous tumor model in immunosuppressed NOD/ SCID mouse. When the tumor in the xenotransplant reached $100 \mathrm{~mm}^{3}$, the animals were treated with $25 \mathrm{mg} / \mathrm{kg}$ of the inhibitor or it vehicle (DMSO) during 5 days for a period of 4 weeks. The animals were then sacrificed and tumor volume and weight were evaluated. Results: HSP90 was positive in 182 tissue samples (33\% intense, $28 \%$ moderate, and $26 \%$ weak) and negative in 27 cases (13\%). HSP90 inhibition in vitro with GA and 17-AAG on cell lines induced cytotoxicity and significantly reduced the cellular migration and the expression of client oncoproteins of HSP90. Treatment with 17-AAG in the subcutaneous tumor significantly reduce the weight and tumoral volume when compared with the control group.

Conclusions: Our results suggest the HSP90 is highly expressed in primary tumors and GBC cell lines. This is the first report of using HSP90 inhibitors as a potential therapeutic alternative in GBC treatment. (Fondecyt 1130204, FONDAP ACCDiS). 
1800 Pancreaticoduodenectomy (PD) Without Pancreatic Adenocarcinoma: A Retrospective Review of 90 Cases Over 10 Years With Cytological and Radiological Correlation in a Subset of Patients With Final Diagnosis of Chronic Pancreatitis

Kavita Varma, Ramya Gadde, Rakesh Varma, Veena Shah. Henry Ford Hospital, Detroit, MI; University of Pittsburgh Medical Centre, Pittsburgh, PA.

Background: Of all the PDs performed for pancreatic neoplasia, in $13.4 \%$ of pancreas no tumor was identified and the final histopathological diagnosis was "Chronic Pancreatitis" (CP). We attempted to correlate the radiologic, cytologic and histopathologic features in this subgroup.

Design: All cases of PDs from June 2001 to December 2011 were searched and cases without pancreatic adenocarcinoma were included and reviewed. Of 261 cases of PD, 35 disclosed CP. Cytologic and radiologic findings were correlated in each case. Results: Total 261 PDs procedures were performed. Ninety cases were identified without the histopathological diagnosis of pancreatic adenocarcinoma. Among this cohort the diagnoses were as follows: chronic pancreatitis (35), intraductal pancreatic mucinous neoplasm (13), pancreatic neuroendocrine tumor (16), serous cystadenoma (6), solid pseudopapillary tumor (4) and others (16). Within chronic pancreatitis group, the cytology and radiological findings are shown in Table 1.

Table 1: Radiological and Cytological Features in Chronic Pancreatitis Patients

\begin{tabular}{|l||c||c||c||c|}
\hline \multicolumn{1}{|c||}{ Radiological Diagnosis $(\mathrm{n}=35)$} & \multicolumn{3}{c|}{ Cytological Diagnosis ( $\mathrm{n}=35)$} \\
\hline \hline & Negative & Suspicious & Positive & $\begin{array}{c}\text { Not } \\
\text { Available }\end{array}$ \\
\hline \hline Mass Lesion $(\mathrm{n}=5)$ & 0 & 0 & 0 & 5 \\
\hline Cystic Lesion $(\mathrm{n}=8)$ & 3 & 0 & 0 & 5 \\
\hline \hline Pancreatobilary duct dilatation $(\mathrm{n}=5)$ & 3 & 1 & 0 & 1 \\
\hline \hline Enlarged Pancreas +/- calcification $(\mathrm{n}=9)$ & 5 & 1 & 0 & 3 \\
\hline \hline Unremarkable Pancreas $(\mathrm{n}=8)$ & 2 & 1 & 2 & 3 \\
\hline
\end{tabular}

Conclusions: Even with available diagnostic modalities, PD procedures performed for presumed tumor resection yielded the postoperative histopathologic diagnosis of "chronic pancreatitis" in a significant number of cases (13. 4\%). In 13 cases with radiologic diagnosis of mass lesion or cystic lesion, no neoplasm was identified in the PD specimen. Distal periductal fibrosis and proximal duct dilatation as well as enlarged pancreas with or without calcifications (14 cases) yielded the diagnosis of chronic pancreatitis as expected. This study gives us a perspective of unneccesay PD with resultant morbidity and mortality, which may be avoidable. Furthur correlated studies are needed to eliminate the $13.4 \%$ PDs without pancreatic adenocarcinoma.

\section{A Novel Concept: Creation of Minimally Invasive Orthotopic Murine} Model of Pancreatic Cancer

Neha Varshney, Jasneet Bhullar, Yaniv Cozacov, Sushant Chaudhary, Jacqueline Tilak, Milessa Decker, Carol Freeman, Vasuki Anandan, Michael Jacobs, Vijay Mittal. University of Toledo Medical Center, Toledo, OH; Providence Hospital and Medical Center, Southfield, MI.

Background: Orthotopic murine models of pancreatic cancer represent an important tool for evaluating pathophysiology, diagnostic techniques and treatment strategies. Several genetically modified mouse tumors and xenograft models have been reported. Genetic models have unpredictable growth \& variable waiting period, while orthotopic models are operative ones, difficult to create and result in irregular metastasis. There is a constant endeavor to create an orthotopic model which replicates the human disease process. Design: Orthotopic pancreatic tumors were induced in 20 SCID mice using a novel technique. Low dose electrocoagulation of pancreas under laparoscopic guidance (using Coloview-mouse colonoscope) with thin electrode, followed by injection of $0.1 \mathrm{cc}$ $\mathrm{BxPC} 3$ pancreatic cancer cells was done ( $\mathrm{n}=12$, study group). Control mice underwent electrocoagulation alone ( $\mathrm{n}=4$, group 1$)$ and tumor cell injection alone $(\mathrm{n}=4$, group 2$)$. Mice were evaluated for tumor growth and metastasis by necropsy ( 4 and 8 weeks for experimental group; 8 weeks for control group).

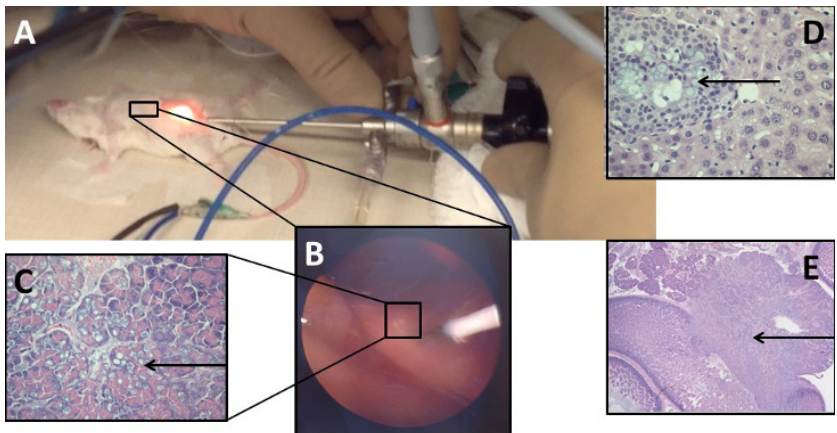

A : SHOWS THE SETUP FOR LAPAROSCOPIC BOVIE INJURY OF PANCREAS

B: SHOWS THE LAPAROSCOPIC VIEW OF BOVIE INJURY OF PANCREAS

C: HISTOPATHOLOGY (H\&E) OF PANCREAS SHOWS INFITERATING ADENOCARCINOMA (MARKER)

D: HISTOPATHOLOGY (H\&E) OF LIVER SHOWING LIVER METASTASIS (MARKER)

E: HISTOPATHOLOGY (H\&E) SHOWING LOCAL BOWEL INVASION OF PANCREATICCARCINOMA
Results: Tumors were detected in 11/12 mice in experimental group, $1 / 4$ in control group 2 , and none in control group 1 . The histopathological features in these murine models were found to be similar to that of human. Over time an increase in tumor growth, tumor volume, lymphovascular invasion of pancreas, with metastasis to lymph nodes and surrounding organs were noted.

Conclusions: We report a novel concept of tumor cell implantation at site of electrocoagulation of pancreas. Combined with the minimally invasive technique, yields a replicative orthotopic murine model of pancreatic cancer. Our model is minimally invasive, easy to create, and overcomes the limitations of the existing models. It will lead to better understanding of pancreatic cancer and will open doors to newer diagnostic and therapeutic tools.

\section{C-MYC Amplification in PDA Is Associated With Poor Outcome} and Adenosquamous Subtype

Megan Wachsmann, Nicholas Borja, Erik Knudsen, Prasad Koduru, Agnes Witkiewicz. The University of Texas Southwestern, Dallas, TX

Background: Pancreatic adenosquamous carcinoma is an uncommon and aggressive pancreatic malignancy that has yet to be fully characterized. C-MYC is a transcription factor that functions in cell growth, differentiation and apoptosis, and is amplified in numerous lymphoid as well as gastrointestinal malignancies but is poorly characterized in pancreatic ductal adenocarcinoma (PDA). Here we evaluated the presence of C-MYC amplifications in PDA using whole exome sequencing (WES) and Fluorescence in situ hybridization (FISH)

Design: Whole exome sequencing was performed on 109 PDA case. TruSeq Exome Enrichment (FC-121-1048) was used to build libraries that were subjected to pairedend whole exome sequencing using Illumina HiSeq2500 platform. Data processing and analysis were performed using Broad Institute Firehose pipeline. To obtain copy number alterations GISTIC 2.0 module version 6.2 with join segment size $=4$ and confidence level $=0.95$ was used. Fluorescence in situ hybridization (FISH) was preformed on selected cases subjected to WES and additional 15 cases of ASC using Vysis LSI MYC dual color "break-apart" rearrangement probe. Increased copy number was defined as 4-5 C-MYC signals per call and amplifications as $>6$ signals per cell. Kaplan-Meier plots were used to evaluate association with survival.

Results: Interrogation of amplified and deleted regions for association with survival revealed that the amplification of the 8q24 region, harboring the MYC oncogene, was associated with poor outcome $(\mathrm{p}=0.0013)$. The amplification of $\mathrm{C}-\mathrm{MYC}$ was confirmed by FISH. C-MYC amplified cases were overrepresented in the adenosquamous subtype of PDA. Analysis of additional 15 cases of adenosquamous carcinoma by FISH revealed, 4 cases with increased C-MYC copy number and 5 cases with amplification. Combined analysis of WES and FISH revealed amplifications in 11 of 27 pancreatic adenosquamous carcinoma cases $(41 \%)$.

Conclusions: These data demonstrate that C-MYC amplifications are common in this particularly aggressive pancreatic cancer subtype and provide a potential therapeutic target in a disease with an otherwise dismal prognosis.

1803 Composition of the Immune Infiltrate in Pancreatic Adenocarcinoma and Survival Impact

Megan Wachsmann, Nicholas Borja, Uthra Balaji, Agnes Witkiewicz. The University of Texas Southwestern, Dallas, TX

Background: Despite extensive efforts, the overall survival for pancreatic ductal adenocarcinoma (PDA) is unchanged. The host immune response, characterized by immune cell infiltrate and their products, is the primary barrier to tumor development and a potential therapeutic target. We investigated the lymphocyte, neutrophil and plasma cell infiltrate in PDA epithelium and stroma. Additionally, we evaluated the immunohistochemical expression of programmed death-ligand 1 (PD-L1), forkhead box P3 (FOXp3) and CD163 and their relationship to clinicopathologic parameters. PD-L1 expression (on tumor epithelial and immune cells) as well as infiltration of FOXP3 expressing $\mathrm{T}$ regulatory cells and CD163 positive M2 macrophages contributes to immune anergy however detailed analysis of these makers has not been simultaneously performed in a large cohort of PDA patients.

Design: Tumor tissue-microarrays with 203 PDA cases annotated with clinicopathologic data were stained for PDL-1, FOXp3 and CD163. Peri-tumoral and intra-tumoral neutrophils, lymphocytes and plasma cells were assessed using whole tissue sections corresponding to TMA cases and scored as absent, mild to moderate or strong based on number of cells. IHC markers were stratified into low and high expression groups based on published criteria. Survival curves were plotted based on individual and combinatorial biomarker status and correlations were determined. Unsupervised, random forest clustering was then performed to determine specific PDA phenotypes and their relationship to prognosis.

Results: PDL1 correlated with a higher tumor grade $\left(\mathrm{p}=2.37 \times 10^{-4}\right)$ however there was no relation of expression to overall survival. Presence of FOXP3 expressing T regulatory cells did not portend worse survival, while infiltrate of CD163 positive M2 macrophages correlated with shorter overall survival (HR 2.438, $\mathrm{p}=0.002$ ). High peri-tumoral neutrophilic infiltrate was noted in PDAs with particularly poor prognosis $(p=0.0455)$, and plasma cell infiltrate trended toward worse survival $(p=0.069)$. No correlation with survival was noted regarding lymphocytic infiltrate. Random forest clustering yielded 3 immune infiltrate defined subtypes. The worse prognosis cluster was characterized by low expression of PDL1, high positivity for CD163 and FOXp3 and had a mean survival of 20 months. In contrast, low expression of PDL1, CD163 and FOXP3 was associated with mean survival 26 months, though these differences did not achieve statistical significance $(\mathrm{p}=0.553)$.

Conclusions: Our findings provide characterization of immune cell infiltrate in PDA and may help in immune based therapies. 


\section{Pancreatic Ductal Adenocarcinoma Is Profoundly Inhibited By} CDK4/6 Inhibitors

Megan Wachsmann, Nicholas Borja, Uthra Balaji, Erik Knudsen, Agnes Witkiewicz. The University of Texas Southwestern Medical Center, Dallas, TX.

Background: Pancreatic ductal adenocarcinoma (PDA) carries a dismal prognosis despite medical intervention. Loss of CDKN2A tumor suppressor occurs frequently in PDA. The CDKN2A gene encodes protein, p16ink4a which is a potent inhibitor of cyclin-dependent kinases 4 and $6(\mathrm{CDK} 4 / 6)$. p16ink4a is dominant to KRAS and can constrain oncogenic proliferation in many contexts. CDK4/6 inhibitors may represent a means to restore the physiological loss of CDKN2A and treat PDA. We utilized three models to investigate the inhibition of CDK4/6 on PDA.

Design: Three models were used to determine the therapeutic efficacy of CDK4/6 inhibition in PDA. First, PDA cell lines were used to delineate the features of response to CDK4/6 inhibition, mechanisms of resistance, and to define novel combination treatments. Second, a primary tumor explant model was employed, since established models may not reflect the complex biology and tumor microenvironment. Tumor slices from 13 clinically resected PDA specimens were cultured on semi-solid support. The explants maintained histologic architecture, biomarker profile, and proliferative index of the primary specimen. They provide the opportunity to delineate response to CDK4/6 inhibition in an unselected patient population. Third, patient-derived xenografts (PDX) of resected disease were utilized to examine the response to CDK4/6 inhibition in vivo. Results: Cell lines had variable responses to CDK4/6 inhibition as a single agent; however, combination therapy with MTOR or MEK inhibitors provided substantia efficacy across all models. This diversity of response was not recapitulated in tumor explants. In this context, treatment with PD-0332991 showed suppression of proliferation across all models, with the exception of single resistant case harboring loss of the RB tumor suppressor. Similarly, patient-derived xenografts exhibited inhibition of Ki-67 proliferation marker and growth suppression. These data indicate that CDK4/6 inhibition is effective in suppressing growth of PDA under diverse physiological contexts.

Conclusions: Our data demonstrates that PDA growth is inhibited by selective targeting of CDK $4 / 6$ in ex-vivo primary tumor explants and patient-derived xenografts. The variable response to PD-0332991 in cultured cell lines and xenografts may reflect a more aggressive phenotype, and potentially the altered biology of models propagated in long term culture. Importantly, the data suggest that CDK4/6 inhibition may be particularly effective in the control of resectable disease given the potent suppression of tumorigenic growth in both explant and PDX models.

1805 Development of BRAF FISH Assay for the Detection of BRAF Gene Rearrangements Identified in Pancreatic Acinar Cell Carcinomas

Lu Wang, Olca Basturk, Juliann Chmielecki, Jeffrey Ross, Philip Stephens, Volkan Adsay, Gokce Askan, David Klimstra. Memorial Sloan Kettering Cancer Center, New York, NY; Foundation Medicine Inc, Cambridge, MA; Albany Medical College, Albany, NY; Emory University, Atlanta, GA.

Background: Pancreatic acinar cell carcinomas (ACCs) account for $\sim 1 \%$ of pancreatic cancers diagnosed annually in the US. Recently, $B R A F$ gene rearrangements were identified in over $20 \%$ of ACCs using next-generation sequencing (NGS)-based platforms. The pilot NGS study revealed eight different $B R A F$ fusions, 5 of which were $S N D 1-B R A F$ with minor differences in the fusion points. Despite a diversity of fusion partners and fusion points, all $B R A F$ rearrangements involved conserved $B R A F$ break points that preserve the kinase domain. Therefore, we developed a dual color, break-apart FISH assay to detect $B R A F$ gene rearrangements in ACCs.

Design: A blinded FISH investigation was performed on the $36 \mathrm{ACCs}$ that had been studied by NGS-based analysis as well as on 2 pancreatoblastomas. The BRAF FISH probe was obtained from Empire Genomics, which is a mixture of two clone contigs hybridizing to the chromosome band $7 \mathrm{q} 34$ flanking $B R A F$ gene locus. The green fluorochrome-labeled probe hybridizes telomeric (5') to $B R A F$; the orange fluorochrome-labeled probe hybridizes centromeric (3') to $B R A F$. BRAF rearrangement may present as either "broken apart" signal or "single orange (3')" signal (missing green signal) which indicates the loss of 5' $B R A F$ but the 3' $B R A F$ coding the kinase domain is preserved.

Results: The FISH study identified $B R A F$ gene rearrangements in $7(19 \%) \mathrm{ACCs}, 6$ of which were positive by NGS study including 3 SNDI-BRAF cases. NGS-based study failed to detect one case which was overtly positive by FISH. Interestingly, all three cases with the fusion partner rather than SND1 showed "single orange (3')" FISH signal pattern; in contrast, "broken-apart" signal was dominant (2/3) for $S N D 1-B R A F$ fusions. $S N D 1$ and $B R A F$ are $\sim 13 \mathrm{Mb}$ apart on genomic DNA, respectively mapping to $7 \mathrm{q} 31$ and $7 \mathrm{q} 34$, and running in opposite directions on chromosome 7. FISH did not reveal $B R A F$ gene rearrangements in the two pancreatoblastomas.

Conclusions: The discovery of recurrent $B R A F$ fusions in ACCs has uncovered a new therapeutic target for patients whose tumors harbor these changes. The BRAF FISH analysis is a time-efficient test and is capable of detecting $B R A F$ gene rearrangements without prior knowledge of 5' fusion partners. Therefore, it can be used to identify ACC patients suitable for targeted therapy using MEK inhibitors.
1806 Senescent Fibroblasts Upregulate Cytokines That Can Increase Pancreatic Cancer Invasion

Tao Wang, Roya Navab, Emin Ibrahimov, Faiyaz Notta, Ming-Sound Tsao. University of Toronto, Toronto, ON, Canada; Princess Margaret Cancer Centre, Toronto, ON, Canada; Ontario Institute for Cancer Research, Toronto, ON, Canada.

Background: Pancreatic ductal adenocarcinoma (PDAC) is a lethal malignancy associated with extensive fibrosis. Cancer associated fibroblasts (CAFs) form a major component of the PDAC microenvironment. Cellular senescence is known to induce a secretory phenotype in fibroblasts. It is unknown if senescent CAFs can alter PDAC behaviour.

Design: CAFs were isolated from collagenase-digested human PDAC and stained by immunofluoresence (IF) to confirm differentiation. CAFs were cultured in 3D collagen gel to assess contractility. Growth rates were measured with the xCelligence system. Senescence was detected by $\beta$-galactosidase assay or by immunohistochemistry (IHC) for $\mathrm{p} 16$ and $\mathrm{Ki67}$. Prolonged culture or $\mathrm{H}_{2} \mathrm{O}_{2}$ was used to induce senescence. In vitro Matrigel invasion assay was performed using a transwell co-culture system with pancreatic cell lines and CAFs +/- a CXCR2 inhibitor. qRT-PCR was performed on genes for various secreted proteins. Conditioned media from CAFs was used for a cytokine ELISA array.

Results: 16 PDAC CAF lines have been isolated. By IF, they expressed CD90, PDGFR $\beta$, vimentin, and aSMA, consistent with a myofibroblastic phenotype. CAF collagen contractility correlated inversely with growth rate $(\mathrm{p}=0.001)$ and correlated positively with the prevalence of senescent cells $(\mathrm{p}=0.004)$. IHC on human PDAC cases revealed 71 of $86(83 \%)$ cases possessed p16+/Ki67- subpopulations of fibroblasts, consistent with senescence. Senescent CAFs expressed higher mRNA and protein levels of several cytokines including IL6, CXCL1, and CXCL8. Conditioned media from senescent CAFs did not affect PDAC cell line growth rates. Senescent CAFs induced 2.5-3.5 fold greater Matrigel invasion by MiaPaca2 $(\mathrm{p}=0.03)$ and $\mathrm{L} 3.6 \mathrm{pl}(\mathrm{p}=0.03)$ compared to control non-senescent CAFs. This was mitigated by inhibiting CXCR2, a receptor for CXCL1 and CXCL8 that is expressed by many pancreatic cancer cell lines.

Conclusions: Senescent CAFs possess a distinct phenotype of halted growth, increased contractility, and upregulated cytokine secretion. Senescent CAFs may play a role in pancreatic cancer cell invasion and their altered pathways may serve as therapeutic targets.

1807 Pseudoinvasion Patterns That Closely Mimic Invasive Carcinoma in Mucinous Cystic Neoplasms of Pancreas and Liver: Histopathologic Differential Diagnostic Criteria Elucidated In an Analysis of 150 Cases

Yue Xue, M Reid, Gizem Akkas, Olca Basturk, Hyejeong Choi, Kee-Taek Jang, Brian Quigley, Alyssa Krasinskas, Volkan Adsay. Emory University School of Medicine, Atlanta, GA; Memorial Sloan Kettering Cancer Center, New York, NY; SMC, Seoul, Korea.

Background: Invasive carcinomas arising from mucinous cystic neoplasms (MCNs) are often tubular type and subtle, and have recently been found to have aggressive behavior. We have observed pseudoinvasive proliferations that can mimic malignancy and be diagnostically challenging.

Design: 102 pancreatic and 48 hepatic MCNs were evaluated for invasive and pseudoinvasive glandular proliferations

Results: Invasive carcinoma was present in 16 cases (11\%). In 52/134 (39\%) noninvasive cases, pseudoinvasive glandular proliferations were noted. While glandular complexity, invagination and tangential cuts could mimic invasion, we identified 4 distinct patterns of pseudoinvasion. (1) Microcystic elongated irregular glands with attenuated cells, often showing neutrophilic/cellular debris in their lumen (MEGAN) were encountered most commonly (30\%). These were multifocal (mean, 3 foci), small (2-3 $\mathrm{mm}$; largest, $18 \mathrm{~mm}$ ), typically noted in the hypercellular areas of ovarian-type stroma (OTS), but also seen forming linear/band-like structures in areas with inflamed OTS. The attenuated, bland cells often showed grooves but had fine nuclear chromatin and grew in a syncytial-pattern. (2) Epithelial clusters lying in clefts in OTS, some with myxoid stromal change, some forming abortive glands, and often showing foamy microvesicular cytoplasm (or paradoxical eosinophilia) with disorganized, atypical nuclei. (3) Small, round tubules, often filled with neutrophilic debris, embedded in an inflamed stroma, with cells exhibiting vesicular nuclei, wrinkled nuclear membranes and grooves but no hyperchromasia. (4) Entrapped irregular glands in fibrotic stroma. Often composed of less than 3-4 glands embedded in either diffuse fibrotic stroma without OTS or in the center of coarse, keloid-like collagen, mimicking corpora albicantia (possibly areas of prior biopsy sites). None of the above pseudoinvasion cases with available clinical follow-up had recurrent/metastatic behavior. In comparison with invasive carcinomas, these pseudoinvasive cases occurred in a younger age group (mean age, 46 vs 55$)$ and had similar tumor sizes $(7.8 \mathrm{~cm}$ vs $7.8 \mathrm{~cm})$

Conclusions: MCNs often show complex glandular patterns that can mimic invasive carcinomas. The recognition of these pseudoinvasive patterns should help distinguish them from invasive carcinoma, which is of great clinical significance.

1808 Suppression of Pancreatic Ductal Carcinoma Growth and Invasion By Targeting MUC13 Mucin Through MicroRNA-145 - A Tumor Heterograft Study in Mice

Nadeem Zafar, Sheema Khan, Stephen Behrman, Meena Jaggi, Subhash Chauhan. University of Tennessee Health Science Center, Memphis, TN.

Background: Pancreatic cancer (PACa) is the $4^{\text {th }}$ leading cause of cancer death in USA Aberrant Mucin 13 (MUC13) expression is highly involved in PACa pathogenesis and appears as a potential target for therapeutic intervention. MicroRNAs have been implicated in cancer initiation and progression via their ability to affect expression of genes and proteins that regulate cell proliferation and/or cell death. Our studies indicate 
that microRNA-145 (miR-145) expression decreases with the progression of PACa, allowing aberrant expression of MUC13. Restitution of lost levels of miR-145 may prevent PACa progression by down-regulating MUC13 expression.

Design: A pilot study using six-week-old athymic $\mathrm{Nu} / \mathrm{nu}$ mice was carried out to investigate the effect of miR-145 restitution. Mice were divided into three treatment groups, control (six mice), non-targeting control mimics (NC, six mice) and miR-145 (six mice). Tumors in mice were implanted by injecting PACa HPAF-II cells, having high MUC13 expression, into the right flank subcutaneously. After the tumors developed, (avg. volume $80 \mathrm{~mm}^{3}$ ) miR-145 mice group was injected intratumorally with miR145 mimics 9 times. Tumor growth was followed for 21 days until the tumors reach 700 $\mathrm{mm}^{3}$. The mice were then euthanized followed by the analysis of tumor growth, volume and metastasis. Tumor volumes were examined as a function of time (discreet), group (control, NC, miR-145) and interaction between them.

Results: We demonstrate thatthe tumor growth and volume regressed drastically with miR-145 replenishment in mice compared to control and NC treated mice. On investigation of mice tumors, we found that the tumors from miR-145 treated mice had drastic reduction in the expression of MUC13 and its target HER2 using in-situ hybridization and immunohistochemistry techniques. This suggested an important role of miR-145 in the inhibition of pancreatic tumor growth by directly targeting MUC13. Conclusions: The current study provides important insights into the tumor suppressor role of miR-145 in a well-known tumor-promoting network that includes MUC13. The study delineates the association of alterations in miR-145 levels with MUC13 expression that may have a potential role in initiation and progression of pancreatic ductal carcinoma. miR-145 induced down-regulation of MUC13 is associated with tumor growth reduction in pancreatic xenograft mice model, highlighting miR-145 as an attractive modulator of MUC-13 in stemming the initiation and growth of PACa.

1809 Morphology and Molecular Evidences of Malignant Potential of Entrapped Ducts in Pancreatic Neuronendocrine Tumors

Wendi Zhou, Jayalakshmi Panicker Balakrishna, Wei Zhang, Abdelsalam Sharabi, Violette Ghali. Mount Sinai St. Luke's - Roosevelt Hospital and Beth Israel Medical Center, New York, NY.

Background: Pancreatic endocrine tumors are well documented for containing entrapped small, cytologically bland ductules. However, pre-neoplastic changes of those entrapped glands on both morphology and molecular levels are not sufficiently investigated.

Design: Total of 25 cases of pancreatic well-differentiated neuronendocrine tumor between 2006 and 2013 were retrospectively analyzed. Pre-neoplastic changes (pancreatic intraepithelial lesions, PanIN) were detected in 9 cases. To investigate whether these PanIN like changes in entrapped glands are preneoplastic or reactive, immunohistochemistry for DPC4, p53, P16, EGFR were performed in all cases. In two cases with PanIN-2 lesions, EGFR gene amplification was analyzed by FISH, followed with mutational analyses for EGFR and KRAS genes using DNA extracted from FFPE tissue after microdissection. Epithelial mucin overexpression was evaluated by staining for MUC1, MUC2, MUC5.

Results: Entrapped ductules were proven to be present in 18 cases by immunohistochemistry staining for CK19, CEA, and CA19.9. Neuronendocrine components were confirmed by staining for synaptophysin and chromogranin. All 9 cases with PanIN changes in entrpped ducts are significantly larger in size (average $4.5 \mathrm{~cm}$, range 3.0-6.6 cm) compared to PanIn negative tumors (average $2.0 \mathrm{~cm}$, range 1.5-3.5 $\mathrm{cm})$. In two cases, entrapped ducts showed extensive proliferation and acquired at least PanIN-2 like changes with profound papillary structures and moderate nuclear atypia. Positive EGFR immunostaining was detected in 6 of 9 PanIN lesions (including two PanIN2 lesions), among which 4 exhibited diffuse $2+$ or $3+$ immunoreactivity. However, EGFR gene amplification was only detected in one PanIN2 lesion. Mutations in K-RAS were detected in both PanIN-2 lesions. No mutations were found in the matched nonlesional ductal areas, suggesting that mutations were acquired during transformation. Immunohistochemically, two PanIN2 lesions expressed MUC1 and MUC5, one PanIN2 lesion had weaker staining of P16. No lesions showed aberrant P53 expression, or loss of DPC4 staining.

Conclusions: Entrapped ducts in pancreatic neuronendocrine tumor can undergo extensive proliferation and acquire PanIN-2 like morphology, apposing challenges in distinguishing from true mixed ductal-endocrine neoplasms. Molecular and immunohistochemical evidences support these lesions to be pre-malignant rather than reactive, and are found in larger size tumors. Further investigation are needed to understand the significance of this pre-malignant changes.

\section{Pathobiology (including Pan-genomic/ Pan-proteomic approaches to cancer)}

1810 Molecular Profiling of Breast Ductal Carcinoma In Situ (DCIS) Gene Expression Signature of HER2 Positive DCIS

Emmanuel Agosto-Arroyo, Tatyana Isayeva, Shi Wei, Jonas Almeida, Shuko Harada. University of Alabama, Birmingham, AL.

Background: Although significant advance has been made in understanding the molecular changes in invasive breast carcinoma (IBC), the exact molecular behavior of DCIS is not well understood. We have shown HER2 overexpression in DCIS is associated with developing IBC. Our hypothesis is that differentially expressed genes in HER2+ DCIS are associated with high risk of developing IBC. The aim of this study is to investigate the differences in gene expression profile between HER2 positive and HER2 negative DCIS
Design: Resection specimens received in 2012-13 with the diagnosis of DCIS without IBC were screened. Based on ER, PR, and HER 2 status, a total of 18 cases with high nuclear grade DCIS [6 ER/PR+, HER2-(ER/PR+), 6 ER/PR-, HER2+(HER2), and 6 ER/PR/HER2-(triple negative; TN)] were selected. RNA was extracted from DCIS areas of the formalin fixed paraffin embedded blocks with 6 non-neoplastic breast tissue controls. Expression array was performed with Affymetrix Human Gene 1.0 ST array at Heflin Center for Genomic Science Core Laboratories. The data was analyzed using the MATLAB software.

Results: A total of 1,682 transcripts were clustered by the specific subtype and the difference of their signal intensity. Thirty genes were identified to be under or overexpressed in the HER2 subtype as compared to the two other groups (Figure 1). Among those, the PROM1 gene was consistently found to be overexpressed in HER2 subtype, as compared to other two subtypes. Expression of this gene is associated with several types of cancer. PEG10 gene was overexpressed in the HER2 group compared to $\mathrm{TN}$ and the control groups.

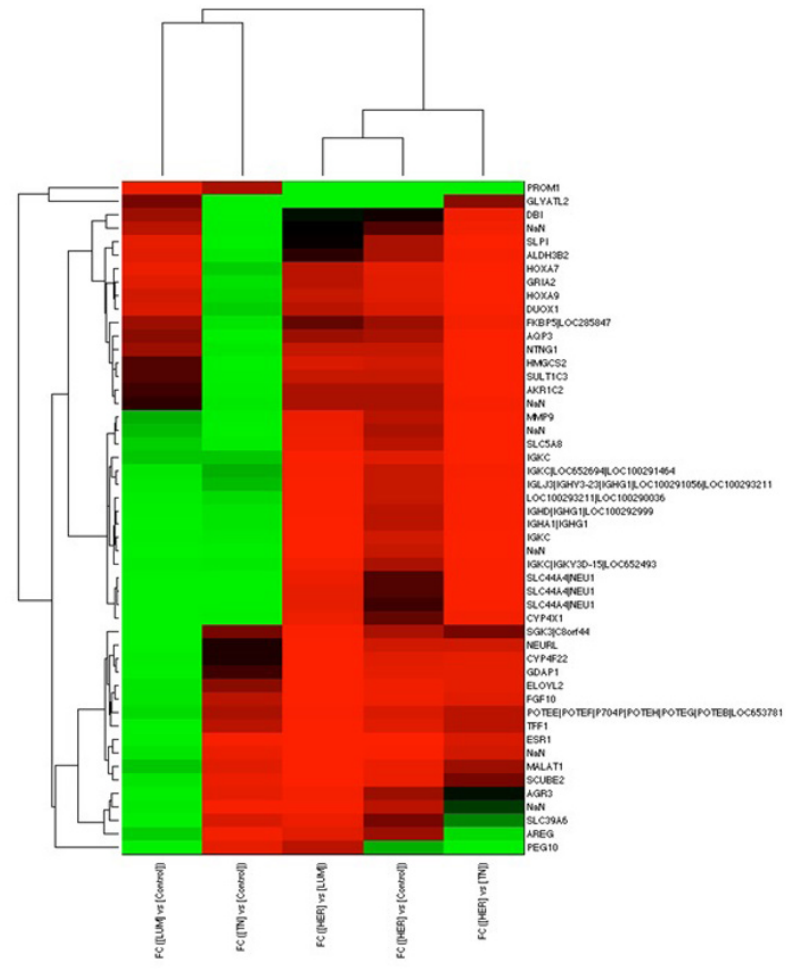

Figure 1. Hierarchical analysis of genes differentially expressed (green-over, red-under) in HER2 subtype compared to control, ER/PR+ (desiganated as LUM) or TN groups. Conclusions: We identified unique gene signatures expressed differently in HER2+ DCIS, which may be associated with development of IBC. The result may enhance our understanding of the mechanisms of breast cancer progression and become the basis for developing new predictive biomarkers and/or therapeutic targets.

1811 Comprehensive Evaluation of DOG1 in Squamous and Urothelial Carcinomas Reveals Frequent Expression Across Anatomic Sites

Deema Alkapalan, Emily McMullen, Andrew Bellizzi. University of Iowa, Iowa City, IA Background: Discovered on GIST1 (DOG1), a calcium-activated chloride channel, is most familiar as a gastrointestinal stromal tumor marker. DOG1 was independently discovered as part of a chromosomal region (11q13) frequently amplified in head and neck squamous cell carcinoma (HNSCC). DOG1 overexpression is prognostically significant in HNSCC, and overexpression correlates with gene amplification. We performed DOG1 immunohistochemistry (IHC) on a cohort of SCCs and urothelial carcinomas (UCs) to determine whether expression was HNSCC-specific or represented a more generalized phenomenon.

Design: DOG1 IHC was performed on tissue microarrays constructed from 243 SCC (anus 22, uterine cervix 25, esophagus 16, larynx 21, lung 28, penis 21, skin 21, ventral tongue 21 , tongue base 11 , tonsil 16 , vagina 19 , vulva 22 ) and 45 UCs (bladder), using polyclonal (Spring) and monoclonal (DOG1.1, Zeta) antibodies. Tumors were arrayed in triplicate. Extent $(\%)$ and intensity $(0,1+, 2+, 3+)$ of staining were assessed and an $\mathrm{H}$-score (extent $\mathrm{x}$ intensity) calculated. $\mathrm{H}$-scores $>0$ were considered positive. Results: DOG1-positivity was noted in 48\% (polyclonal) and 26\% (monoclonal) of 288 squamotransitional carcinomas (STCs). 2 or $3+$ positivity was seen in $22 \%$ (polyclonal) and $13 \%$ (monoclonal). Detailed expression data are presented in the Table. 